\title{
HYPERBOLIC CONVEX CORES AND SIMPLICIAL VOLUME
}

\author{
PETER A. STORM
}

\begin{abstract}
AвSTRACT. This paper investigates the relationship between the topology of hyperbolizable 3-manifolds $M$ with incompressible boundary and the volume of hyperbolic convex cores homotopy equivalent to $M$. Specifically, it proves a conjecture of Bonahon stating that the volume of a convex core is at least half the simplicial volume of the doubled manifold $D M$, and this inequality is sharp. This paper proves that the inequality is in fact sharp in every pleating variety of $\mathrm{AH}(M)$.
\end{abstract}

\section{INTRODUCTION}

Let $M$ be a compact oriented 3-manifold with nonempty incompressible boundary such that the interior of $M$ admits a complete convex cocompact hyperbolic metric. Define the topological invariant

$$
\mathcal{V}(M):=\inf \left\{\operatorname{Vol}\left(C_{N}\right) \mid N \text { hyperbolic \& homeomorphic to } \operatorname{int}(M)\right\},
$$

where $C_{N}$ is the convex core of $N$. Bonahon conjectured that $\mathcal{V}(M)$ equals half the simplicial volume of the doubled manifold $D M$ (see also [13, Sec.7]). This paper proves Bonahon's conjecture.

The beginning of this paper proves the following Kleinian version of the BessonCourtois-Gallot theorem [5].

Theorem 1.1. If $N$ is a hyperbolic 3-manifold homotopy equivalent to $M$ then

$$
\operatorname{Vol}\left(C_{N}\right) \geq \frac{1}{2} \operatorname{Simp} \operatorname{Vol}(D M) .
$$

Moreover, if $\operatorname{Vol}\left(C_{N}\right)=\frac{1}{2} \operatorname{Simp} \operatorname{Vol}(D M)>0$ then $M$ is acylindrical, $N$ is convex cocompact, and $\partial C_{N}$ is totally geodesic.

(See Theorems 3.1 and 3.2) Note that $\operatorname{Vol}\left(C_{N}\right)=0$ if and only if $N$ is either a Fuchsian group or an extended Fuchsian group. (An extended Fuchsian group is a degree two extension of a Fuchsian group.) The main tool used to prove Theorem 1.1 is due to Souto 28, building on work of Besson-Courtois-Gallot 5 .

Let us postpone an introduction of simplicial volume to Section 2.6. Briefly, if $X$ is a closed oriented 3-manifold for which Thurston's geometrization conjecture is true, then it is possible to cut $X$ along essential spheres and tori into manifolds admitting one of Thurston's eight geometries. It is known the simplicial volume of $X$ is the total volume of the hyperbolic pieces of this decomposition of $X$. Simplicial volume also known as the Gromov norm. (They differ by a constant.)

Date: August 22nd, 2005.

This research was partially supported by a National Science Foundation Postdoctoral Fellowship and the Clay Mathematics Institute Liftoff program.

2000 Mathematics Subject Classification: 53C25, 57N10. 
In its second half, this paper proves the inequality of Theorem 1.1 is sharp. In fact, using the work of Bonahon-Otal [7, the inequality is proven to be sharp in every pleating variety of the deformation space.

Theorem 5.1. Let $\lambda$ be the bending measured lamination on $\partial M$ of the boundary of the convex core of a convex cocompact hyperbolic manifold $N$ homeomorphic to the interior of $M$. Then for $0<\varepsilon<1$ there exists a convex cocompact hyperbolic manifold $N_{\varepsilon}$ homeomorphic to the interior of $M$ with bending measured lamination $\varepsilon \lambda$ such that

$$
\operatorname{Vol}\left(C_{N_{\varepsilon}}\right) \longrightarrow \frac{1}{2} \operatorname{Simp} \operatorname{Vol}(D M) \quad \text { as } \quad \varepsilon \rightarrow 0
$$

(The existence of the manifolds $N_{\varepsilon}$ above follows quickly from the work of BonahonOtal [7. The central point of Theorem 5.1 is ( $\dagger)$.) Together, Theorems 1.1 and 5.1 solve the above conjecture. Namely,

Corollary 1.2. $\mathcal{V}(M)=\frac{1}{2} \operatorname{Simp} \operatorname{Vol}(D M)$. In particular, $\mathcal{V}$ is a homotopy invariant of hyperbolizable compact oriented 3-manifolds with incompressible non-toroidal boundary.

(See Corollary 2.8.) A pared version of Corollary 1.2 is also proved.

Acknowledgements: The author thanks Richard Canary for his essential assistance at every stage of this research. Juan Souto's doctoral thesis is a fundamental and essential tool in this paper. The author thanks him for visiting the University of Michigan and sharing some of his ideas. This research benefited from conversations with Ian Agol, Cyril Lecuire, and Caroline Series. Much of this manuscript was prepared during a stays at the Fourier Institute in Grenoble and the Isaac Newton Institute in Cambridge. The author thanks his hosts for their generous hospitality.

1.1. Outline. The two main tools used to prove Theorem 1.1 are an inequality of Souto [27. Sec.3,Thm.4], and a previous result of the author [30, Thm.8.1]. For convenience, we restate them here. For a geometrizable closed 3-manifold $X, X_{\text {hyp }}$ is the finite volume hyperbolic manifold obtained from the geometric decomposition of $X$.

Theorem 1.3. [28, Sec.3,Thm.4] (Souto's inequality) If $X$ is a closed geometrizable Riemannian 3-manifold, then

$$
h(\tilde{X})^{3} \operatorname{Vol}(X) \geq 2^{3} \operatorname{Vol}\left(X_{\text {hyp }}\right)=2^{3} \operatorname{Simp} \operatorname{Vol}(X),
$$

where $h(\tilde{X})$ is the volume growth entropy of the universal cover of $X$.

(Souto also proved a related rigidity theorem. See [28.) Let $M$ be a hyperbolizable compact connected oriented 3-manifold with incompressible boundary. Let $\mathcal{I}(M)$ be the set of isometry classes of hyperbolic 3-manifolds homotopy equivalent to $M$. A geometrically finite hyperbolic 3-manifold $N$ has Fuchsian ends if each component of $N-C_{N}$ has Fuchsian holonomy. It is a consequence of Thurston's Geometrization Theorem and Mostow Rigidity that $M$ is acylindrical if and only if there exists a unique convex cocompact $N_{g} \in \mathcal{I}(M)$ with Fuchsian ends (Corollary 2.1).

Theorem 1.4. [30, Thm.8.1] Let $M$ be a compact acylindrical 3-manifold. Let $N_{g} \in \mathcal{I}(M)$ be the unique convex cocompact manifold with Fuchsian ends. Then for all $N \in \mathcal{I}(M)$,

$$
\operatorname{Vol}\left(C_{N}\right) \geq \operatorname{Vol}\left(C_{N_{g}}\right)
$$


with equality if and only if $N$ and $N_{g}$ are isometric.

We now outline the contents of Sections 3]5. (Section 2 is devoted to preliminaries.) Define the constant

$$
\mathcal{V}:=\frac{1}{2} \operatorname{SimpVol}(D M)
$$

Section 3: For $N \in \mathcal{I}(M)$ we first prove that $\operatorname{Vol}\left(C_{N}\right) \geq \mathcal{V}$. The rough idea is to double $C_{N}$ to form $D C_{N}$, smoothly approximate the resulting metric space by Riemannian manifolds $A_{i}^{\infty}$, and apply Souto's inequality to the pair $A_{i}^{\infty}$ and $D M$. Next, the rigidity statement of Theorem 1.1 is proven. Namely, if $N \in \mathcal{I}(M)$ and $\operatorname{Vol}\left(C_{N}\right)=\mathcal{V}>0$, then $N$ is convex cocompact with Fuchsian ends, and $M$ is acylindrical. For this outline suppose that $N$ is convex cocompact. Assume $M$ is not acylindrical. Then $D M$ is not hyperbolizable, and consequently $(D M)_{\text {hyp }}$ is a noncompact collection of finite volume hyperbolic manifolds. By 22, there is a sequence of Riemannian metrics $g_{n}$ on $D M$ converging geometrically to $(D M)_{\text {hyp. In }}$ particular, the diameter of $\left(D M, g_{n}\right)$ is going to infinity. We may use the barycenter method of Besson-Courtois-Gallot and a technique of Souto to produce uniformly Lipschitz surjections $D C_{N} \longrightarrow\left(D M, g_{n}\right)$. Since $D C_{N}$ is compact, this is a contradiction. Therefore $M$ is acylindrical. An application of Theorem 1.4 completes the proof.

Section 4: This section collects results from the literature about how continuously convex cores vary under geometric convergence, and the behavior of convex core volumes under geometric convergence. Results due to Bowditch [8], McMullen [23, and Taylor 31 are used. These facts are later used to prove Theorem 5.1

Section 5: This section proves Theorem 5.1. In particular, we find a sequence of convex cocompact manifolds $N_{n} \in \mathcal{I}(M)$ such that $\operatorname{Vol}\left(C_{N_{n}}\right) \rightarrow \frac{1}{2} \operatorname{SimpVol}(D M)$. For this, the idea is to take manifolds $N_{n}$ such that the bending measure on $\partial C_{N_{n}}$ is a fixed projective measured lamination with total measure going to zero. Such

manifolds were proven to exist by Bonahon-Otal [7. Some work is required to prove that these "lightly pleated" manifolds converge in some sense to a (disconnected) manifold with Fuchsian ends, and that convex core volume tends toward the expected value $\mathcal{V}$.

\section{PRELIMINARIES}

This section collects the most important definitions for this paper.

2.1. Pared 3-manifolds [25, Def.4.8]. Let $M$ be a compact orientable irreducible 3 -manifold with nonempty boundary. Assume no component of $M$ is a 3-ball. Let $P \subseteq \partial M$ be a closed subset. $(M, P)$ is a pared 3 -manifold if the following three conditions hold.

(1) Every component of $P$ is an incompressible torus or a compact annulus.

(2) Every noncyclic abelian subgroup of $\pi_{1}(M)$ is conjugate into the fundamental group of a component of $P$.

(3) Every $\pi_{1}$-injective cylinder $C:\left(S^{1} \times I, S^{1} \times \partial I\right) \longrightarrow(M, P)$ is relatively homotopic to a map $\psi$ such that $\psi\left(S^{1} \times I\right) \subseteq P$. 
The submanifold $P \subset \partial M$ is the paring locus of $(M, P)$. By Thurston's Geometrization Theorem [25], $(M, P)$ is a pared 3-manifold if and only if there exists a geometrically finite hyperbolic structure on the interior of $M$ such that $C_{N} \cong M-P$.

Many standard topological notions have natural adaptations to the pared category. For example, a pared homotopy equivalence is (coincidentally) a homotopy equivalence of pairs. $(M, P)$ has pared incompressible boundary if the inclusion map $\partial M-P \hookrightarrow M$ is $\pi_{1}$-injective on all components. An annulus $C: S^{1} \times I \longrightarrow M$ is essential in $(M, P)$ if $C$ is $\pi_{1}$-injective, $C$ is a map of pairs

$$
C:\left(S^{1} \times I, S^{1} \times \partial I\right) \longrightarrow(M, \partial M-P),
$$

and $C$ is not homotopic rel boundary into $\partial M[34, \mathrm{pg} .244] .(M, P)$ is pared acylindrical if $(M, P)$ has pared incompressible boundary and no essential annuli. The double $D(M, P)$ of a pared manifold across its boundary is the compact manifold with toroidal boundary components obtained by gluing two copies of $M$ together along $\overline{\partial M-P}$. If $(M, P)$ is pared acylindrical then by Thurston's Geometrization Theorem [25], $D(M, P)$ admits a complete finite volume hyperbolic metric on its interior.

2.2. The characteristic submanifold. This paper will make extensive use of the characteristic submanifold theory developed by Jaco, Johannson, and Shalen in [17, 18, 16. For a brief discussion of this theory from the point of view of Kleinian groups, the author recommends Morgan's article [25, pg.88]. For convenience we here define some of the important terminology.

Let $M$ be a compact oriented irreducible Haken 3-manifold. Let $B \subset \partial M$ be an incompressible subsurface. There exists a subpair $(\Sigma, S) \subset(M, B)$ with the following properties (see [25, 17, 18]):

(1) $\Sigma, S \subset M$ are (possibly disconnected) submanifolds and $S \subset \partial \Sigma \cap B$.

(2) Each component of $(\Sigma, S)$ is either a 3-manifold pair of the form ( $I$-bundle, $\partial I-$ subbundle) or a Seifert fibered manifold in which the corresponding components of $S$ are foliated by fibers.

(3) The frontier of $\Sigma$ in $M$ is a collection of essential annuli and tori in $(M, B)$.

(4) No component of $(\Sigma, S)$ is homotopic in $(M, B)$ into another component.

(5) Any essential annulus or torus in $(M, B)$ is homotopic in $(M, B)$ into $(\Sigma, S)$. Moreover, any pair satisfying these conditions is isotopic rel $B$ to $(\Sigma, S)$. By a slight abuse of notation, $(\Sigma, S)$ is called the characteristic submanifold of $(M, B)$.

Now assume $(M, P)$ is a pared manifold with pared incompressible boundary. The characteristic submanifold of $(M, P)$ as a pared manifold is defined to be the characteristic submanifold of $(M, \overline{\partial M-P})$ as a pair. In this case the Seifert fibered components of $\Sigma$ are either solid tori or thickened tori. A component $L$ of the complement of the characteristic submanifold $(\Sigma, S)$ of $(M, P)$ is either a solid torus, a thickened torus, or $(L, \operatorname{Fr} \Sigma \cap L)$ is pared acylindrical.

The window of $(M, P)$ is an $I$-bundle formed as follows. Begin with the $I$ bundle components of the characteristic submanifold $\Sigma$ of $(M, P)$. Now add a regular neighborhood of every essential annulus in the frontier of $\Sigma$ which is not on the boundary of an $I$-bundle component of $\Sigma$. Each of these regular neighborhoods can be viewed as an $I$-bundle with its $\partial I$-subbundle in $S \subset \partial M$. Some of these new $I$-bundles may be homotopic rel $\partial M-P$ into other $I$-bundles. So eliminate any redundant $I$-bundles. The resulting collection of $I$-bundles is the window of 
$(M, P)$ [35, [13, Sec.5.3]. The window is an $I$-bundle over a surface in $M$ called the window base.

2.3. Kleinian Deformation theory. Let $0<\mu_{3}<1$ be a Margulis constant for hyperbolic 3-manifolds. Then for a hyperbolic 3-manifold $N$, the $\mu_{3}$-thin part of $N$ is a disjoint union of bounded Margulis tubes and unbounded cusps [3]. After possibly making $\mu_{3}$ smaller, we may also assume that the ends of $\partial C_{N}$ are totally geodesic in the $2 \mu_{3}$-thin part of $N$ [25, Lem.6.9]. Define $N^{o}$ to be $N$ minus the unbounded components of its $\mu_{3}$-thin part. In other words, $N^{o}$ is the manifold with boundary obtained by removing the cusps from $N$. For $\varepsilon<\mu_{3}, N^{\geq \varepsilon}$ (resp. $N^{\leq \varepsilon}$ ) will denote the closed $\varepsilon$-thick (resp $\varepsilon$-thin) parts of $N$.

Let $M$ be any manifold. Define the deformation set $\mathrm{H}(M)$ as follows. For an oriented hyperbolic 3-manifold $N$ and a homotopy equivalence $m: M \longrightarrow N$, the pair $(N, m)$ is in the set $\mathrm{H}(M)$. The pairs $\left(N_{1}, m_{1}\right)$ and $\left(N_{2}, m_{2}\right)$ are equivalent in $H(M)$ if there exists an orientation preserving isometry $\iota: N_{1} \longrightarrow N_{2}$ such that $\iota \circ m_{1} \sim m_{2}$.

We will also need a relative version of the above set of hyperbolic structures. So now let $(M, P)$ be a pared 3-manifold. Define the deformation set $\mathrm{H}(M, P)$ as follows. For a hyperbolic 3-manifold $N$ and a map $m: M \longrightarrow N^{o},(N, m) \in$ $\mathrm{H}(M, P)$ if there exists a union $Q_{N}$ of components of $\partial N^{o}$ such that $m:(M, P) \longrightarrow$ $\left(N^{o}, Q_{N}\right)$ is a relative homotopy equivalence. $\left(N_{1}, m_{1}\right)=\left(N_{2}, m_{2}\right)$ in $\mathrm{H}(M, P)$ if there exists an orientation preserving isometry $\iota: N_{1} \longrightarrow N_{2}$ such that $\iota \circ m_{1} \sim m_{2}$. $(N, m)$ is minimally parabolic if $Q_{N}=\partial N^{o}$.

Let $\mathcal{I}(M, P)$ be the set of (unoriented) isometry classes of hyperbolic 3-manifolds in $\mathrm{H}(M, P)$.

A hyperbolic manifold $N$ has Fuchsian ends if it is geometrically finite and the components of $N-C_{N}$ have Fuchsian holonomy. If a connected $N$ has Fuchsian ends, then either $C_{N}$ or $\partial C_{N}$ is a totally geodesic subsurface of $N$.

For precision, it will be convenient to consider hyperbolic manifolds equipped with a framed basepoint. A framed basepoint $\omega$ of a hyperbolic manifold $N$ is a point $* \in N$ together with an orthonormal basis for the tangent space $T_{*} N$. A framed manifold will be a pair $(N, \omega)$. By fixing for once and for all a framed basepoint $\omega_{\mathbb{H}^{3}}$ for hyperbolic 3-space, there is a canonical locally isometric covering map

$$
\iota:\left(\mathbb{H}^{3}, \omega_{\mathbb{H}^{3}}\right) \longrightarrow(N, \omega) .
$$

This covering canonically determines a faithful representation

$$
\rho: \pi_{1}(N, *) \longrightarrow \operatorname{Isom}^{+}\left(\mathbb{H}^{3}\right) \cong \mathrm{PSl}_{2} \mathbb{C} .
$$

We will use the notation $\pi_{1}(N, \omega)$ to denote the subgroup of $\mathrm{PSl}_{2} \mathbb{C}$ given by the image of $\rho$.

A particularly striking property of pared acylindrical 3-manifolds is the following corollary of Thurston's Geometrization Theorem, Mostow rigidity, and Waldhausen's Theorem.

Corollary 2.1. [33, pg.14] Let $(M, P)$ be a pared acylindrical 3-manifold. Then there exists a unique minimally parabolic $N_{g} \in \mathcal{I}(M, P)$ with Fuchsian ends.

2.4. The bending measured lamination. Let $(M, P)$ be a pared 3-manifold with pared incompressible boundary. Let $N \in \mathcal{I}(M, P)$ be a minimally parabolic 
geometrically finite hyperbolic 3-manifold equipped with a homeomorphism

$$
h: M-P \longrightarrow C_{N} \text {. }
$$

The geometry of the boundary of the convex core $C_{N}$ is a pleated surface, and it is described by a measured geodesic lamination on $\partial C_{N}$ 14. Pull back this measured lamination via $h$ to a measured lamination on $\partial M-P$. This measured lamination $\beta$ on $\partial M-P$ is the bending measured lamination of the hyperbolic manifold $N$ (homeomorphically) marked by $h$.

In Section 5 we will need the following theorem, whose proof follows from the work of Bonahon [6], Bonahon-Otal [7, Kerckhoff-Hodgson [15, and Lecuire [21.

Theorem 2.2. Retain the notation of the previous paragraph. Pick $0<\varepsilon<1$ and consider the measured lamination $\varepsilon \beta$ on $\partial M-P$. There exists a minimally parabolic geometrically finite $N_{\varepsilon} \in \mathcal{I}(M, P)$ equipped with a homeomorphism

$$
h_{\varepsilon}: M-P \longrightarrow C_{N_{\varepsilon}}
$$

such that:

(1) $\varepsilon \beta$ is the bending measured lamination of $N_{\varepsilon}$ marked by $h_{\varepsilon}$, and

(2) The volumes $\operatorname{Vol}\left(C_{N_{\varepsilon}}\right)$ are non-increasing as $\varepsilon \rightarrow 0$.

Let $p \subset P \subset \partial M$ be an embedded 1-manifold which is a deformation retract of the annular components of $P$. Make $p$ into a measured lamination on $\partial M$ by assigning weight $\pi$ to each component of $p$. In order to apply [7, 21, we need

Lemma 2.3. Let $\lambda$ be a measured lamination on $\partial M-P$. Consider the following three conditions on $\lambda$ :

(a) Each closed leaf of $\lambda$ has weight less than or equal to $\pi$.

(b) There exists an $\eta>0$ such that if $A$ is an essential annulus of $(M, P)$ then $i(\partial A, \lambda) \geq \eta$.

(c) If $D$ is an essential disk of $M$ ( $M$ may have compressible boundary!), then $i(\lambda \cup p, \partial D)>2 \pi$.

If $\lambda$ satisfies conditions (a), (b), and (c) and $0<\varepsilon<1$, then $\varepsilon \lambda$ satisfies conditions (a), (b), and (c).

In the proof of Lemma 2.3 we will need

Lemma 2.4. If $D$ is an essential disk of $M$ then $\partial D \subset \partial M$ intersects intersects $p$ in at least two points.

Proof of Lemma 2.4: Suppose $\partial D$ intersects $p$ in a single point. Let $\mathcal{N} p$ (resp. $\mathcal{N} D$ ) be a regular neighborhood of $p$ (resp. $D$ ) in $M$. The frontier of $\mathcal{N} p \cup \mathcal{N} D$ is a compressing disk of $M$ which does not intersect $p$. Since $(M, P)$ is pared incompressible, this is a contradiction.

Proof of Lemma 2.3; Suppose $\lambda$ is a measured lamination of $\partial M-P$ satisfying conditions (a), (b), and (c). Then $\varepsilon \lambda$ immediately satisfies condition (a). Condition (b) is satisfied for the constant $\varepsilon \cdot \eta$. Finally, let $D$ be an essential disk of $M$. By Lemma 2.4 $i(p, \partial D) \geq 2 \pi$. Condition (c) applied to $\lambda$ implies that either $i(p, \partial D)>2 \pi$ or $i(\lambda, \partial D)>0$. Therefore

$$
i((\varepsilon \lambda) \cup p, \partial D)=\varepsilon i(\lambda, \partial D)+i(p, \partial D)>2 \pi .
$$


Proof of Thm. 2.2: The proof is broken into two cases.

In the first case let us assume that the support of the measured lamination $\beta$ is a union of simple closed curves. Then conclusion (1) of Theorem 2.2 follows from Lemma 2.3 and [7, Thm.2]. It follows from [7, Thm.3] that the manifolds $N_{\varepsilon}$ are the unique manifolds satisfying conclusion (1). By [15, Thm.4.7], we may choose the $N_{\varepsilon}$ and framings $\omega_{\varepsilon}$ such that the path

$$
\begin{aligned}
\eta:(0,1) & \longrightarrow \operatorname{Hom}\left(\pi_{1}(M), \mathrm{PSl}_{2} \mathbb{C}\right) \\
\varepsilon & \longmapsto\left\{\rho_{\varepsilon}: \pi_{1}(M) \longrightarrow \pi_{1}\left(N_{\varepsilon}, \omega_{\varepsilon}\right)\right\}
\end{aligned}
$$

is smooth. Since the path $\eta$ is smooth, a version of the Schläfli formula due to Bonahon [6, Cor.2] implies that the volume $\operatorname{Vol}\left(C_{N_{\varepsilon}}\right)$ is strictly decreasing as $\varepsilon \rightarrow 0$.

The second case is the general case. The main tool is [21, Thm.A]. Using Lemma [2.3. we may apply [21, Thm.A] (and its proof in [21, Sec.5.1]) to the lamination $\varepsilon \beta \cup p$. From this we may conclude that there exists a sequence of measured laminations $\beta^{j}$ converging to $\beta$ in the space of measured laminations on $\partial M-P$ such that the following are true for all $0<\varepsilon \leq 1$ :

- The support of $\beta^{j}$ is a union of simple closed curves.

- There exists a sequence of geometrically finite hyperbolic manifolds $N_{\varepsilon}^{j}$ with bending measured lamination $\varepsilon \beta^{j}$ and a homeomorphism $h_{\varepsilon}^{j}: M-P \longrightarrow C_{N_{\varepsilon}^{j}}$.

- For each $\varepsilon>0$, any subsequence of the sequence $\left\{\left(N_{\varepsilon}^{j}, h_{\varepsilon}^{j}\right)\right\}_{j}$ has a further subsequence which converges algebraically to a manifold satisfying conclusion (1) of Theorem 2.2 .

By a diagonalization argument we can pass to a subsequence $\left\{j_{k}\right\}$ such that for all rational $\varepsilon \in(0,1)$ the sequence $\left\{\left(N_{\varepsilon}^{j_{k}}, h_{\varepsilon}^{j_{k}}\right)\right\}_{k}$ converges algebraically to a manifold $\left(N_{\varepsilon}, h_{\varepsilon}\right)$ satisfying conclusion (1) of Theorem 2.2. For each irrational $\varepsilon \in(0,1)$ define $\left(N_{\varepsilon}, h_{\varepsilon}\right)$ to be an accumulation point of the sequence $\left\{\left(N_{\varepsilon}^{j_{k}}, h_{\varepsilon}^{j_{k}}\right)\right\}_{k}$. To simplify the notation, let us re-index the subsequence $\left\{j_{k}\right\}$ by the natural numbers, which we will again denote by simply $\{j\}$.

The geometrically finite manifolds $\left(N_{\varepsilon}, h_{\varepsilon}\right)$ and $\left(N_{\varepsilon}^{j}, h_{\varepsilon}^{j}\right)$ are minimally parabolic in $\mathrm{H}(M, P)$. Therefore any algebraically convergent subsequence of $\left(N_{\varepsilon}^{j}, h_{\varepsilon}^{j}\right)$ satisfies the property: $h_{\varepsilon}(\gamma) \in \mathrm{PSl}_{2} \mathbb{C}$ is parabolic if and only if $h_{\varepsilon}^{j}(\gamma)$ is parabolic for all $j$. Such an algebraically convergent sequence is said to be type-preserving. In the setting of geometrically finite manifolds, it is known that type-preserving algebraically convergence sequences converge strongly. (A more general theorem is proven by Anderson-Canary in [2][Thm.3.1]. Without explicit mention, this issue of strong convergence is carefully studied in [7] [Ch.2].)

By the first case in this proof, the manifolds $N_{\varepsilon}^{j}$ are uniquely determined and

$$
\varepsilon<\varepsilon^{\prime} \quad \text { implies } \operatorname{Vol}\left(C_{N_{\varepsilon}^{j}}\right)<\operatorname{Vol}\left(C_{N_{\varepsilon^{\prime}}^{j}}\right) \text {. }
$$

Pick a rational number $t \in\left(\varepsilon, \varepsilon^{\prime}\right)$. By the previous paragraph, the manifold $\left(N_{\varepsilon}, h_{\varepsilon}\right)$ is a strong accumulation point of the sequence $\left\{\left(N_{\varepsilon}^{j}, h_{\varepsilon}^{j}\right)\right\}$, and for each $j$ we have the inequality

$$
\operatorname{Vol}\left(C_{N_{\varepsilon}^{j}}\right)<\operatorname{Vol}\left(C_{N_{t}^{j}}\right)
$$

Convex core volume is continuous in the strong topology 31. Therefore

$$
\operatorname{Vol}\left(C_{N_{\varepsilon}}\right) \leq \operatorname{Vol}\left(C_{N_{t}}\right)
$$

Applying an identical argument to $\varepsilon^{\prime}$ shows that

$$
\operatorname{Vol}\left(C_{N_{\varepsilon}}\right) \leq \operatorname{Vol}\left(C_{N_{\varepsilon^{\prime}}}\right) .
$$


This proves part (2) of Theorem 2.2. (The extra argument in this case is necessary because the author does not know how to find a smooth path of deformations analogous to $\eta$. Without such a smooth path, Bonahon's version of the Schläfli formula cannot be applied.)

2.5. Lightly bent curves in hyperbolic 3 -manifolds. In Section 5 it will be necessary to compare the length of a lightly bent essential closed curve on the boundary of the convex core with the length of the curve's geodesic representative. To do so we use the following estimate due to Lecuire [21] (proved also by Series).

Lemma 2.5. 21, Lem.A.1] Let $N$ be a geometrically finite hyperbolic 3-manifold. Let $\beta$ be the bending measured lamination on the boundary of the convex core $C_{N}$. Let $c \subset \partial C_{N}$ be a closed curve which is a geodesic in the intrinsic hyperbolic metric on $\partial C_{N}$. If it exists, let $c^{*}$ be the closed geodesic of $N$ freely homotopic to $c$. For $\varepsilon<\pi / 2$ there exist constants $K_{\varepsilon}, A_{\varepsilon}$ such that: if $i(c, \beta) \leq \varepsilon$ then

$$
\ell_{M}(c) \leq K_{\varepsilon} \cdot\left(\ell_{M}\left(c^{*}\right)+A_{\varepsilon}\right),
$$

where $K_{\varepsilon} \rightarrow 1$ and $A_{\varepsilon} \rightarrow 0$ as $\varepsilon \rightarrow 0$, and $\ell_{M}\left(c^{*}\right):=0$ when $c$ is not homotopic to a closed geodesic.

2.6. Simplicial volume. For detailed definitions and an introduction to simplicial volume in the context of 3-manifolds, the author recommends [32, Ch.6].

Let $X$ be a closed oriented 3-manifold. The ostensibly simple definition of simplicial volume is the following.

$$
\operatorname{SimpVol}(X)=V_{3} \inf \left\{\sum_{i}\left|c_{i}\right|\right\},
$$

where the infimum is taken over all singular 3 -chains $\sum_{i} c_{i}$ realizing the fundamental class of $X$ in singular homology, and $V_{3} \approx 1.01$ is the supremal volume of a hyperbolic simplex in $\mathbb{H}^{3}$. The simplicial volume divided by $V_{3}$ is known as the Gromov norm. There is a similar definition of simplicial volume for compact 3-manifolds whose boundary consists of tori (see [32, Ch.6]). These definitions will not be used directly in this paper. It will be more useful to use the relation between simplicial volume and hyperbolic volume which we now describe.

Let $X$ now be a compact oriented irreducible (possibly disconnected) 3-manifold with (possibly empty) boundary equal to a collection of incompressible tori. Assume $X$ is geometrizable, meaning $X$ can be cut along embedded essential tori into pieces which admit a geometric structure locally modelled on one of the eight Thurston geometries. Let $X_{\text {hyp }}$ be the finite volume (possibly disconnected) complete hyperbolic manifold obtained from the hyperbolic pieces of the geometric decomposition of $X$. In this situation there is the following useful theorem of Thurston/Soma relating simplicial volume to hyperbolic volume.

Theorem 2.6. 27] [32, Ch.6] Let $X$ be as above. The simplicial volume of $X$ is the volume of $X_{\text {hyp }}$, i.e.

$$
\operatorname{Simp} \operatorname{Vol}(X)=\operatorname{Vol}\left(X_{\text {hyp }}\right) \text {. }
$$

In particular, if a (possibly disconnected) manifold $X^{\prime}$ is obtained by cutting $X$ along an essential embedded torus, then

$$
\operatorname{Simp} \operatorname{Vol}(X)=\operatorname{Simp} \operatorname{Vol}\left(X^{\prime}\right) .
$$


Let $(M, P)$ be a compact oriented irreducible (possibly disconnected) pared 3manifold with pared incompressible boundary. Let $\Sigma$ be the characteristic submanifold of $(M, P)$. Let $\left(M_{\text {acyl }}, Q\right)$ be the compact (not necessarily connected) pared acylindrical 3-manifold obtained from the disjoint union of the pared acylindrical pieces of $M-\Sigma$. By Corollary 2.1, there exists a geometrically finite minimally parabolic hyperbolic 3-manifold $N_{g} \in \mathcal{I}\left(M_{\text {acyl }}, Q\right)$ homeomorphic to $\operatorname{int}\left(M_{\text {acyl }}\right)$ such that the boundary of the convex core $C_{N_{g}}$ is totally geodesic. (Keep in mind that neither $M_{\text {acyl }}$ nor $N_{g}$ is necessarily connected.)

Proposition 2.7. $(D(M, P))_{\text {hyp }}$ is isometric to $D C_{N_{q}}$. In particular, the simplicial volume of the double $D(M, P)$ is twice the volume of $C_{N_{g}}$.

Proof: $\quad$ One can verify that the inclusion map $D\left(M_{\text {acyl }}, Q\right) \hookrightarrow D(M, P)$ is $\pi_{1^{-}}$ injective. Almost by definition, the complement $D(M, P)-D\left(M_{\text {acyl }}, Q\right)$ can be cut along embedded essential tori into Seifert fiber spaces. Therefore $(D(M, P))_{\text {hyp }}$ is homeomorphic to the interior of $D\left(M_{\text {acyl }}, Q\right)$, which is in turn homeomorphic to $D C_{N_{g}}$. The proposition now follows from Mostow rigidity and Theorem 2.6.

Corollary 2.8. Simp $\operatorname{Vol}(D(M, P))$ is a pared homotopy invariant in the category of compact oriented irreducible pared manifolds with pared incompressibly boundary.

Proof: By Johannson's deformation theorem [18, the pared homeomorphism type of the complement of the characteristic submanifold is a pared homotopy invariant in the above category.

Roughly speaking, drilling curves out of a 3-manifold cannot decrease its simplicial volume. We shall need a precise formulation of this. The following inequality was proved by Agol, completing a sketch in [32, Ch.6].

Proposition 2.9. [1] Let $X$ be a compact oriented irreducible geometrizable 3manifold with (possibly empty) boundary consisting of incompressible tori. If $\gamma \subset X$ is a compact embedded 1-manifold with a regular neighborhood $\mathcal{N} \gamma$, and $X-\mathcal{N} \gamma$ is hyperbolizable then

$$
\operatorname{Simp} \operatorname{Vol}(X-\mathcal{N} \gamma)>\operatorname{Simp} \operatorname{Vol}(X) \text {. }
$$

In [1], Proposition 2.9 is stated for $X$ without boundary, but the proof goes through unchanged for manifolds with toroidal boundary components. In the setting of pared manifolds, we have

Corollary 2.10. If $(M, P)$ and $(M, Q)$ are pared 3-manifolds with pared incompressible boundary, and $P \subseteq Q$ is a collection of connected components, then

$$
\operatorname{Simp} \operatorname{Vol}(D(M, Q)) \geq \operatorname{Simp} \operatorname{Vol}(D(M, P)) \text {. }
$$

Proof: A manifold homeomorphic to $D(M, P)$ can be produced by appropriately gluing solid tori onto the boundary of $D(M, Q)$. The corollary then follows from [32, Prop.6.5.2].

2.7. Volume growth entropy. Let $X$ be a geodesic metric space of Hausdorff dimension $n, \widetilde{X}$ be the universal cover of $X$, and $\mathcal{H}^{n}$ be $n$-dimensional Hausdorff measure. The volume growth entropy of $\widetilde{X}$ is the number

$$
h(\widetilde{X}):=\limsup _{R \rightarrow \infty} \frac{1}{R} \log \mathcal{H}^{n}\left(B_{\widetilde{X}}(x, R)\right),
$$


where $x$ is any point in $\widetilde{X}$, and the ball $B_{\tilde{X}}(x, R)$ is in $\widetilde{X}$.

The following inequality will be used in Section 3

Theorem 2.11. 11, pg.40] If $X$ is an Alexandrov space with curvature bounded below by -1 and Hausdorff dimension $n$, then the volume growth entropy of $\widetilde{X}$ is less than or equal to the volume growth entropy of $\mathbb{H}^{n}$. In other words

$$
h(\widetilde{X}) \leq h\left(\mathbb{H}^{n}\right)=n-1 .
$$

2.8. Smoothing and geometrically doubling the convex core. In Section 3 it will be necessary to consider smooth approximations of the convex core. Here we establish some convenient notation and facts. The geometric structure theory presented in Morgan's article [25, Sec.6] (in particular [25, Lem6.8]) will be used repeatedly.

Suppose $N$ is a geometrically finite hyperbolic 3 -manifold. Then $C_{N}$ has finite volume. For $\varepsilon>0$, let $\mathcal{N}_{\varepsilon} C_{N}$ be an $\varepsilon$-neighborhood of $C_{N}$. By [14, Lem.1.3.6], the boundary of $\mathcal{N}_{\varepsilon} C_{N}$ is $\mathcal{C}^{1,1}$-smooth.

For $\delta>0$, let $C_{N, \delta} \subset N$ be a closed convex submanifold of $N$ such that

$$
C_{N} \subset \operatorname{int}\left(C_{N, \delta}\right) \subset C_{N, \delta} \subset \mathcal{N}_{\delta} C_{N},
$$

$\operatorname{Vol}\left(C_{N, \delta}\right)<\operatorname{Vol}\left(C_{N}\right)+\delta, \partial C_{N, \delta}$ is $\mathcal{C}^{1,1}$-smooth, and the unbounded components of $\partial C_{N, \delta} \cap N \leq \mu_{3}$ are totally geodesic. One can find such a submanifold by beginning with $\mathcal{N}_{\varepsilon} C_{N}$ (for some $\varepsilon<\delta$ ) and smoothly tapering the ends of $\partial \mathcal{N}_{\varepsilon} C_{N}$ so that the ends of the boundary of the tapered manifold are totally geodesic in the $\mu_{3}$-thin part of $N$. (There are many possible submanifolds satisfying these conditions. It will not matter which is chosen.)

We may metrically double $C_{N, \delta}$ across its boundary to obtain the path metric space $D C_{N, \delta}$. Since $\partial C_{N, \delta}$ is $\mathcal{C}^{1,1}$, the doubled manifold $D C_{N, \delta}$ is a $\mathcal{C}^{1,1}$-manifold without boundary. The Riemannian metric on $N$ induces a Riemannian metric tensor on $D C_{N, \delta}$, but on a $\mathcal{C}^{1,1}$ manifold it only makes sense to ask whether or not the resulting metric tensor is Lipschitz. The induced metric tensor is Lipschitz on each half of the doubled convex core, and glues continuously across the boundary. (Verify this using coordinates in the form of a $\mathcal{C}^{1,1}$ product neighborhood of $\partial C_{N, \delta}$.) The resulting metric tensor on $D C_{N, \delta}$ is therefore Lipschitz. Moreover, outside a compact set $D C_{N, \delta}$ is a hyperbolic rank 2 cusp. This follows because, by definition, the boundary of $C_{N, \delta}$ is totally geodesic outside a compact set. (See the previous paragraph for the definition of $C_{N, \delta}$.) We note that $D C_{N, \delta}$ is an Alexandrov space with curvature bounded below by -1 [29, Lem.5.4] (see also [26, Thm.5.2]).

Since we are working in dimension three, the $\mathcal{C}^{1,1}$-structure on $D C_{N, \delta}$ contains a unique $\mathcal{C}^{\infty}$ structure. So $D C_{N, \delta}$ admits the structure of a smooth manifold equipped with a Lipschitz Riemannian metric. It is convenient to approximate the Lipschitz metric tensor on $D C_{N, \delta}$ by a smooth Riemannian metric. A standard smooth approximation argument yields the following lemma, whose proof we omit.

Lemma 2.12. Let $(M, g)$ be a smooth manifold equipped with a Lipschitz Riemannian metric tensor. Pick $L>1$. There exists a smooth Riemannian metric $h$ on $M$ such that the identity map

$$
(M, g) \longrightarrow(M, h)
$$

is L-Lipschitz. 


\section{Applying the BARYCENTER MEthod}

Let $(M, P)$ be a connected pared 3-manifold with pared incompressible boundary. To avoid trivial cases, we assume that $\operatorname{Simp\operatorname {Vol}}(D(M, P))>0$. Let $\Sigma$ be the characteristic submanifold of $(M, P)$. Let $\left(M_{\text {acyl }}, Q\right)$ be the compact (not necessarily connected) pared acylindrical 3-manifold obtained from the disjoint union of the pared acylindrical pieces of $M-\Sigma$. By Corollary 2.1, there exists a geometrically finite minimally parabolic hyperbolic 3-manifold $N_{g} \in \mathcal{I}\left(M_{\text {acyl }}, Q\right)$ homeomorphic to $\operatorname{int}\left(M_{\text {acyl }}\right)$ such that the boundary of the convex core $C_{N_{g}}$ is totally geodesic. (Keep in mind that neither $M_{\text {acyl }}$ nor $N_{g}$ is necessarily connected.) Recall that by Proposition 2.7 $\frac{1}{2} \operatorname{Simp} \operatorname{Vol}(D(M, P))=\operatorname{Vol}\left(C_{N_{g}}\right)$. The inequality $\operatorname{SimpVol}(D(M, P))>0$ implies that $\left(M_{\text {acyl }}, Q\right)$ is not empty.

The goal of this is section is to prove the following two theorems.

Theorem 3.1. Let $(M, P)$ and $\left(N_{g}, m_{g}\right)$ be as above. If $N \in \mathcal{I}(M, P)$ then

$$
\operatorname{Vol}\left(C_{N}\right) \geq \operatorname{Vol}\left(C_{N_{g}}\right)=\frac{1}{2} \operatorname{Simp} \operatorname{Vol}(D(M, P)) .
$$

Theorem 3.2. Let $(M, P)$ and $\left(N_{g}, m_{g}\right)$ be as above. If $N \in \mathcal{I}(M, P)$ and

$$
\operatorname{Vol}\left(C_{N}\right)=\operatorname{Vol}\left(C_{N_{g}}\right)=\frac{1}{2} \operatorname{Simp} \operatorname{Vol}(D(M, P))
$$

then $(M, P)$ is pared acylindrical and $N$ is minimally parabolic.

At the end of this section, Theorem 1.1 will be proven using Theorems 3.1 and 3.2

Recall there is a natural way to double the pared manifold $(M, P)$ across $\overline{\partial M-P} \subset$ $\partial M$ to obtain a compact manifold $D(M, P)$ with boundary a disjoint collection of tori (see Section 2.1). Also double the pared manifold $\left(M_{\text {acyl }}, Q\right)$ across its boundary to obtain $D\left(M_{\text {acyl }}, Q\right)$. The doubled manifold $D\left(M_{\text {acyl }}, Q\right)$ is hyperbolizable (see Section 2.1). Double the compact manifold $D(M, P)$ across its boundary to obtain the closed manifold $D D(M, P)$. (To handle all cases simultaneously, we adopt the convention that the double $D X$ of a closed manifold $X$ is simply two disjoint copies of $X$.) From Proposition 2.7 it follows that $(D D(M, P))_{\text {hyp }}$ is homeomorphic to two disjoint copies of $\operatorname{int}\left(D\left(M_{\text {acyl }}, Q\right)\right)$.

Pick a hyperbolic 3 -manifold $N$ in $\mathcal{I}(M, P)$. Without a loss of generality we may assume that $N$ is geometrically finite. Let us temporarily assume that $N$ is minimally parabolic. This assumption will be removed later. Since $\operatorname{SimpVol}(D(M, P))$ is a pared homotopy invariant in the category of pared manifolds with pared incompressible boundary (Corollary 2.8), we may assume there exists a homeomorphism

$$
g:(M, P) \longrightarrow\left(C_{N} \cap N^{\geq \zeta}, C_{N} \cap \partial N^{\geq \zeta}\right)
$$

for some small $\zeta<\mu_{3}$ (see Section 2.3). The constant $\zeta$ is to remain fixed for the remainder of this section.

Metrically double $C_{N, \delta}$ across its boundary to obtain the path metric space $D C_{N, \delta}$. (The following facts are discussed in Section 2.8.) $D C_{N, \delta}$ is a smooth manifold equipped with a Lipschitz metric tensor. Outside of a compact set $D C_{N, \delta}$ is a rank 2 hyperbolic cusp. There is a homeomorphism $D(M-P) \longrightarrow D C_{N, \delta}$. To control the volume growth entropy of the universal cover of $D C_{N, \delta}$, we need the fact that $D C_{N, \delta}$ is an Alexandrov space with curvature bounded below by -1 .

To apply Souto's machinery, we must be working with compact spaces. If $D C_{N, \delta}$ is not compact, the following lemma will allow us to make a compact space out of 
$D C_{N, \delta}$. Its proof is similar to the proof of [22, Prop.2.3] (see also [4] and [28, Prop.8]).

Lemma 3.3. Let $(T \times[0, \infty), g)$ be a rank 2 hyperbolic cusp, where $T$ is a 2 -torus. Pick $\varepsilon>0$. There exists an $R_{\varepsilon}>0$ and a Riemannian manifold $\left(T \times[-1,1], g_{\varepsilon}\right)$ such that:

(1) The sectional curvature of $g_{\varepsilon}$ is between $-1-\varepsilon$ and 0 .

(2) The diffeomorphic involution of $T \times[-1,1]$ taking $(x, t)$ to $(x,-t)$ is a $g_{\varepsilon^{-}}$ isometry.

(3) There is an isometric homeomorphism $\left(T \times\left[0, R_{\varepsilon}\right], g\right) \longrightarrow\left(T \times[-1,-1 / 2], g_{\varepsilon}\right)$.

(4) $\operatorname{Vol}\left(T \times[-1 / 2,1 / 2], g_{\varepsilon}\right)<\varepsilon$.

Moreover, $\lim _{\varepsilon \rightarrow 0} R_{\varepsilon}=\infty$.

Recall that $\partial C_{N, \delta}-N \geq \zeta$ is a (possibly disconnected) totally geodesic surface (Section 2.8). For any $\eta<\zeta$ (where $\zeta<\mu_{3}$ is the constant fixed above), let $D C_{N, \delta}^{\geq \eta}$ indicate the compact manifold with horospherical boundary obtained by "doubling" the $\eta$-thick part $C_{N, \delta} \cap N \geq \eta$ of $C_{N, \delta}$. Outside a compact set, $D C_{N, \delta}$ is a smooth hyperbolic rank 2 cusp. Therefore by using the above lemma there exists a sequence of path metric spaces $A_{i}$ homeomorphic to $D D(M, P)$ such that:

(a) $A_{i}$ is an Alexandrov space with curvature bounded below by $-1-\frac{1}{i}$.

(b) $A_{i}$ is a $\mathcal{C}^{1,1}$-manifold with a Lipschitz Riemannian metric tensor.

(c) There are two isometric embeddings $\phi_{i}^{k}: D C_{N, 1 / i}^{\geq 1 / i} \longrightarrow A_{i}($ for $k=1,2)$ with disjoint images.

(d) $\operatorname{Vol}\left(A_{i}\right) \longrightarrow 2 \operatorname{Vol}\left(D C_{N}\right)=4 \operatorname{Vol}\left(C_{N}\right)$.

Note that if $C_{N}$ is compact, then $A_{i}$ is simply two disjoint copies of $D C_{N, 1 / i}$.

By Theorem 2.11 and property (a) above, $\lim \sup h\left(\widetilde{A_{i}}\right) \leq h\left(\mathbb{H}^{3}\right)=2$. As a final approximation, let $A_{i}^{\infty}$ be a $\mathcal{C}^{\infty}$-smooth Riemannian manifold equipped with $(1+1 / i)$-bilipschitz homeomorphism $\psi_{i}: A_{i} \rightarrow A_{i}^{\infty}$. (Such a space $A_{i}^{\infty}$ exists by Lemma 2.12.) Then also

$$
\lim \sup h\left(\widetilde{A_{i}^{\infty}}\right) \leq h\left(\mathbb{H}^{3}\right)=2 .
$$

We are now prepared to quickly prove Theorem 3.1 .

Proof of Thm.3.1: By Souto's theorem [28, Sec.3,Thm.4],

$$
h\left(\widetilde{A_{i}^{\infty}}\right)^{3} \cdot \operatorname{Vol}\left(A_{i}^{\infty}\right) \geq 2^{3} \operatorname{Vol}\left((D D(M, P))_{\text {hyp }}\right)=2^{3} \cdot 2 \operatorname{Vol}\left(D C_{N_{g}}\right) .
$$

Taking $i \rightarrow \infty$ and dividing both sides of the inequality by $2^{5}$ yields

$$
\operatorname{Vol}\left(C_{N}\right) \geq \operatorname{Vol}\left(C_{N_{g}}\right) \text {. }
$$

This proves Theorem 3.1 under the additional assumption that $N$ is minimally parabolic. It remains to remove this assumption.

Recall the homeomorphism

$$
g:(M, P) \longrightarrow\left(C_{N} \cap N^{\geq \zeta}, C_{N} \cap \partial N^{\geq \zeta}\right) .
$$

Using this homeomorphism, we see that by enlarging the paring locus $R \supset P$, $N$ becomes minimally parabolic in the deformation set $\mathcal{I}(M, R)$ (see Section 2.3). Enlarging the paring locus of $(M, P)$ does not decrease the simplicial volume of the double $D(M, P)$ (Corollary 2.10). This proves the desired inequality in the general case. 
We now prove Theorem 3.2

Proof of Thm 3.2: Assume that $\operatorname{Vol}\left(C_{N}\right)=\operatorname{Vol}\left(C_{N_{g}}\right)$.

To begin, we need the following weak form of Ahlfors regularity for $A_{i}^{\infty}$, which follows from the Bishop-Gromov inequality for Alexandrov spaces [10, Thm.10.6.6]. Recall that for $k \in\{1,2\}$ and $i \in \mathbb{N}$ we have bilipschitz embeddings

$$
\psi \circ \phi_{i}^{k}: D C_{N, 1 / i}^{\geq 1 / i} \longrightarrow A_{i}^{\infty},
$$

where the bilipschitz constant is decreasing to 1 as $i \rightarrow \infty$.

Lemma 3.4. There exists a constant $a>0$ such that for all $r<1, i \gg 0, k \in\{1,2\}$, and $x \in D C_{N, 1 / i}^{\geq \zeta}$ we have

$$
\operatorname{Vol}\left(B_{A_{i}^{\infty}}\left(\psi_{i} \circ \phi_{i}^{k}(x), r\right) \geq a r^{3} .\right.
$$

Proof: Using the bilipschitz embeddings $\psi \circ \phi_{i}^{k}: D C_{N, 1 / i}^{\geq \zeta} \longrightarrow A_{i}^{\infty}$, it suffices to prove the lemma for $D C_{N, 1 / i}^{\geq \zeta}$. Since $D C_{N, 1 / i}^{\geq \zeta}$ is compact, the volume of a unit radius ball in $D C_{N, 1 / i}^{\geq \zeta}$ has a positive lower bound which is uniform for $i \gg 0$. Using this, one may apply the Bishop-Gromov inequality [10, Thm.10.6.6] to obtain the desired result. (Alternatively, the Bishop-Gromov inequality can be avoided by directly considering the geometry of $C_{N, 1 / i}$.)

We now need the following version of a theorem of Leeb [22, Prop.2.3,Prop.2.6] (see also [28, Prop.8] and [4). Recall that $D(M, P)$ is the compact manifold with (possibly empty) toroidal boundary obtained by doubling $M$ along $\overline{\partial M-P}$, and $D D(M, P)$ is the closed manifold obtained by doubling $D(M, P)$.

Theorem 3.5. 22] (see also [28, Prop.8]) $D D(M, P)$ admits a family of metrics $\rho_{i}(i \in \mathbb{N})$ with sectional curvature between $-1-\frac{1}{i}$ and 0 such that:

(1) There is an open $\pi_{1}$-injective subset $V \subseteq D D(M, P)$ homeomorphic to $(D D(M, P))_{\text {hyp }}$ such that: for all $i$ there is an isometric embedding

$$
\left(\mathcal{N}_{i} V, \rho_{i}\right) \longrightarrow(D D(M, P))_{h y p}=D C_{N_{g}} \coprod D C_{N_{g}},
$$

where $\mathcal{N}_{i} V$ is the neighborhood of radius $i$ around $V$ with respect to the metric $\rho_{i}$. Moreover $D D(M, P)$ has a characteristic submanifold disjoint from $V$.

(2) One can choose a basepoint in each component of $V$ and $(D D(M, P))_{\text {hyp }}$ such that the Riemannian manifolds

$$
\left(V, \rho_{i}\right) \text { converge to }(D D(M, P))_{\text {hyp }} \stackrel{\text { isom. }}{=} D C_{N_{g}} \coprod D C_{N_{g}}
$$

in the pointed Gromov-Hausdorff topology.

(3) $\lim \operatorname{Vol}\left(D D(M, P), \rho_{i}\right)=\lim \operatorname{Vol}\left(V, \rho_{i}\right)=4 \operatorname{Vol}\left(C_{N_{g}}\right)$.

The above theorem says the closed manifold $D D(M, P)$ admits non-positively curved metrics which, in the hyperbolizable pieces of $D D(M, P)$, look more and more like hyperbolic manifolds. Moreover, the bits of $D D(M, P)$ which are not hyperbolizable become very long and thin, with small total volume.

We will use a version of the barycenter method due to Souto.

Theorem 3.6. 28, App.A,Prop.5] There exists a sequence of $\mathcal{C}^{1}$ homotopy equivalences $F_{i}: A_{i}^{\infty} \longrightarrow\left(D D(M, P), \rho_{i}\right)$ such that:

(1) There is a sequence $c_{i} \searrow 1$ such that $\left|J a c F_{i}\right| \leq c_{i}$ on $F_{i}^{-1}(V)$. 
(2) There exist constants $\varepsilon, R, r$, and $L$ such that: if $x \in \widetilde{A_{i}^{\infty}}$ is such that the ball $B\left(F_{i}(x), R\right) \subset\left(D D(M, P), \rho_{i}\right)$ is hyperbolic, and $\left|J a c F_{i}(x)\right| \geq(1-\varepsilon) \cdot c_{i}$, then $F_{i}$ is L-Lipschitz on the ball $B_{A_{i}^{\infty}}(x, r)$.

Roughly speaking, the maps $F_{i}$ are nearly volume non-increasing on the bits of $A_{i}^{\infty}$ which map into hyperbolic pieces of $\left(D D(M, P), \rho_{i}\right)$. Moreover, if the map does not dramatically shrink volume at some hyperbolic point in the target, then the map is uniformly Lipschitz. These two properties can be played off each other to show that the maps $F_{i}$ are uniformly Lipschitz on compact sets. The following argument is similar to [28, Sec.3.1] and [5, Ch.7].

Lemma 3.7. There exists an $i_{0}$ such that for all $i \geq i_{0}$ the map

$$
F_{i} \circ \psi_{i} \circ \phi_{i}^{j}: D C_{N, 1 / i}^{\geq \zeta} \longrightarrow\left(D D(M, P), \rho_{i}\right)
$$

is 2 L-Lipschitz, where $j \in\{1,2\}$.

Proof: Without a loss of generality, we may assume that $r<1$ in Theorem 3.6. We have established the limits

$$
\operatorname{Vol}\left(A_{i}^{\infty}\right) \longrightarrow 4 \operatorname{Vol}\left(C_{N}\right) \text { and } \operatorname{Vol}\left(D D(M, P), \rho_{i}\right) \longrightarrow 4 \operatorname{Vol}\left(C_{N_{g}}\right) .
$$

The map $F_{i}$ is nearly volume nonincreasing on the set $F_{i}^{-1}(V)$. From this and conclusion (3) of Theorem 3.5 it follows that,

$$
\left(\operatorname{Vol}\left(A_{i}^{\infty}\right)-\operatorname{Vol}\left(F_{i}^{-1}(V)\right)\right) \longrightarrow 0 .
$$

If $F_{i}$ uniformly shrunk a large piece of $A_{i}^{\infty}$, then too much volume would be lost to hit all of $\left(V, \rho_{i}\right)$ with the nearly volume nonincreasing map $F_{i}$. In other words,

$$
\operatorname{Vol}\left(\left\{x \in A_{i}^{\infty}|\quad| \operatorname{Jac} F_{i}(x) \mid<(1-\varepsilon)\right\}\right) \longrightarrow 0 .
$$

So we've shown that the set of points where $F_{i}$ shrinks volume by a definite amount is small, and the set of points where we do not control the behavior of $F_{i}$ is small. However, these small volume sets may be spread all through the space $A_{i}^{\infty}$. This is where Lemma 3.4 comes in: for $x \in D C_{N, 1 / i}^{\geq \zeta}$, a metric $r$-ball about the point $\psi_{i} \circ \phi_{i}^{k}(x) \in A_{i}^{\infty}$ has volume at least $a r^{3}>0$. So for $i$ sufficiently large, every point $\psi_{i} \circ \phi_{i}^{k}(x) \in A_{i}^{\infty}$ is at most distance $r$ from a point in the (good) set

$$
F_{i}^{-1}(V) \bigcap\left\{x \in A_{i}^{\infty}|| \operatorname{Jac} F_{i}(x) \mid \geq(1-\varepsilon)\right\} .
$$

Therefore for $i \gg 0$ an $r$-neighborhood of the set

$$
F_{i}^{-1}(V) \bigcap\left\{x \in A_{i}^{\infty}|| \operatorname{Jac} F_{i}(x) \mid \geq(1-\varepsilon)\right\}
$$

contains the subspace

$$
\left(\psi_{i} \circ \phi_{i}^{1}\left(D C_{N, 1 / i}^{\geq \zeta}\right)\right) \bigcup\left(\psi_{i} \circ \phi_{i}^{2}\left(D C_{N, 1 / i}^{\geq \zeta}\right)\right) \subseteq A_{i}^{\infty} .
$$

( $\zeta>0$ is the fixed constant from the beginning of the section.) From conclusion (2) of Theorem 3.6 we may conclude that for $i \gg 0$, the maps

$$
F_{i} \circ \psi_{i} \circ \phi_{i}^{j}: D C_{N, 1 / i}^{\geq \zeta} \longrightarrow\left(D D(M, P), \rho_{i}\right)
$$

are $2 L$-Lipschitz, where $j \in\{1,2\}$. This proves the lemma.

With these geometric facts, we can now use a simple topological argument to prove that $(M, P)$ is pared acylindrical. 
Suppose $(M, P)$ is not pared acylindrical. Let $B \subset M$ be an essential annulus. Pick a homotopically nontrivial connected closed curve $\gamma \subset M$ which intersects $B$ essentially. Recall the homeomorphism

$$
g:(M, P) \longrightarrow\left(C_{N} \cap N^{\geq \zeta}, C_{N} \cap \partial N^{\geq \zeta}\right) .
$$

Without a loss of generality, we may assume that $g(\gamma)$ is a smooth finite length curve in $C_{N}$. Now consider $\gamma$ as a curve in $D D(M, P)$. Since $\gamma \subset M$ intersects $B \subset M$ essentially, $\gamma \subset D D(M, P)$ intersects the torus $D B \subset D D(M, P)$ essentially. The complement $\overline{D D(M, P)-V} \subset D D(M, P)$ contains a characteristic submanifold of $D D(M, P)$, implying $D B$ is homotopic into $\overline{D D(M, P)-V}$. Therefore $\gamma$ intersects both $\overline{D D(M, P)-V}$ and $V$ essentially. This implies the $\rho_{i}$-length of the geodesic homotopic to $\gamma$ is going to infinity. This contradicts the fact that the map (1) is $2 L$ Lipschitz and $g(\gamma)$ has finite length. Therefore $(M, P)$ must be pared acylindrical.

To complete the proof of Theorem 3.2 it remains to prove that $N$ must be minimally parabolic. Suppose that $N \in \mathcal{I}(M, P)$ is not minimally parabolic. As in the proof of Theorem 3.1 by enlarging the paring locus $R \supset P, N$ becomes minimally parabolic in $\mathcal{I}(M, R)$ (see Section 2.3). Since by hypothesis $\operatorname{Vol}\left(C_{N}\right)=\frac{1}{2} \operatorname{Simp} \operatorname{Vol}(D(M, P))$, Theorem 3.1 implies that $\operatorname{SimpVol}(D(M, P))=$ $\operatorname{Simp} \operatorname{Vol}(D(M, R))$. Therefore the above argument applied to $N$ in $\mathcal{I}(M, R)$ proves that $(M, R)$ is pared acylindrical. Thus $D(M, R)$ is hyperbolizable. But Proposition 2.9 then implies that $\operatorname{SimpVol}(D(M, R))>\operatorname{SimpVol}(D(M, P))$. This is a contradiction. Therefore, $N$ must be minimally parabolic.

We can now prove

Theorem 1.1. Let $M$ be a compact oriented 3-manifold with nonempty incompressible boundary such that the interior of $M$ admits a complete convex cocompact hyperbolic metric. If $N$ is a hyperbolic 3-manifold homotopy equivalent to $M$ then

$$
\operatorname{Vol}\left(C_{N}\right) \geq \frac{1}{2} \operatorname{Simp} \operatorname{Vol}(D M) .
$$

Moreover, if $\operatorname{Vol}\left(C_{N}\right)=\frac{1}{2} \operatorname{Simp} \operatorname{Vol}(D M)>0$ then $M$ is acylindrical, $N$ is convex cocompact, and $\partial C_{N}$ is totally geodesic.

Proof: The inequality follows from Theorem 3.1. So suppose

$$
\operatorname{Vol}\left(C_{N}\right)=\frac{1}{2} \operatorname{Simp} \operatorname{Vol}(D M)>0 \text {. }
$$

Then by Theorem 3.2, $M$ is acylindrical and $N$ is convex cocompact. It follows from Theorem 1.4 that $\partial C_{N}$ is totally geodesic.

\section{Geometric convergence of Convex cores}

This section will assemble facts from the literature concerning geometric convergence of convex cores. These facts will be used in Section 5.

We begin with a proposition which follows quickly from the following fact: Kleinian limit sets move continuously in the geometric topology when the injectivity radius at the basepoint is uniformly bounded from above and below [20] 23, Prop.2.4].

Lemma 4.1. Let $\left(N_{n}, \omega_{n}\right)$ be a sequence of framed hyperbolic 3-manifolds converging geometrically to $(N, \omega)$. Assume all framed basepoints $\omega_{n}$ (resp $\left.\omega\right)$ are contained 
in the intersection of the convex core $C_{N_{n}}\left(\right.$ resp $\left.C_{N}\right)$ and the $\mu_{3}$-thick part $N_{n}^{\geq \mu_{3}}$ (resp. $\mathrm{N}^{\geq \mu_{3}}$ ). Suppose the convex cores $C_{N_{n}}$ are all homeomorphic, $C_{N_{n}}$ has incompressible boundary, and there is a uniform upper bound on the volume of $C_{N_{n}}$. Then the limit sets $L_{n}$ of $\left(N_{n}, \omega_{n}\right)$ converge in the Hausdorff topology to the limit set $L$ of $(N, \omega)$.

(The framed manifold $\left(N_{n}, \omega_{n}\right)$ canonically determines a subgroup $\pi_{1}\left(N_{n}, \omega_{n}\right) \subset$ $\mathrm{PSl}_{2} \mathbb{C}$. This notation is defined in Section 2.3 . The above hypotheses are not optimal. We prove only what we will need later.)

Proof: Using [23, Prop.2.4], it suffices to prove there is an $R>0$ such that for any $n$ and any point $x \in C_{N_{n}}$, the injectivity radius of $N_{n}$ at $x$ is less than $R$. The uniform upper bound on the volume of the convex cores $C_{N_{n}}$ yields a uniform upper bound $r_{1}$ on the radius of an embedded ball contained in $C_{N_{n}}$. Since the manifolds $N_{n}$ are all homeomorphic, it follows from the Gauss-Bonnet theorem that there is a uniform upper bound $r_{2}$ on the injectivity radii of the surfaces $\partial C_{N_{n}} \subset N_{n}$. Using the fact that $C_{N_{n}}$ has incompressible boundary, it follows that the injectivity radius at a point $x \in C_{N_{n}}$ is less than $r_{1}+r_{2}$.

We combine this with the work of Bowditch [8] and Taylor [31, Thm.7.2] to obtain

Proposition 4.2. Assume the hypotheses of Proposition 4.1. Then the framed submanifolds $\left(C_{N_{n}}, \omega_{n}\right)$ converge in the pointed Gromov-Hausdorff topology to $\left(C_{N}, \omega\right)$.

Proof: By Proposition 4.1, the limit sets $L_{n}$ of $\left(N_{n}, \omega_{n}\right)$ converge in the Hausdorff topoogy to the limit set $L$ of $(N, \omega)$. By [31, Thm.7.2], it follows that for any $r>0$ the submanifolds $\left(C_{N_{n}} \cap B\left(\omega_{n}, r\right)\right)$ converge in the (basepoint free) GromovHausdorff topology to $\left(C_{N} \cap B(\omega, r)\right)$. (In the notation of [31, Thm.7.2], we know that $\Lambda$ is equal to the full limit set of the geometric limit $\Gamma$. With this, the desired convergence is proven in the second paragraph of the proof of [31, Thm.7.2], which in turn uses a result from 8 .

We now generalize the geometric topology slightly to handle sequences of manifolds which are pulling apart into multiple components.

Definition 4.3. (Geometric convergence) Let $\left(N_{n},\left\{\omega_{n}^{i}\right\}_{i=1}^{k}\right)$ be a sequence of hyperbolic 3-manifolds equipped with $k$ framed basepoints $\left\{\omega_{n}^{1}, \ldots \omega_{n}^{k}\right\}$ contained in the intersection $C_{N_{n}} \cap N^{\geq \mu_{3}}$. $\left(N_{n},\left\{\omega_{n}^{i}\right\}\right)$ converges geometrically to a hyperbolic 3-manifold $\left(X,\left\{\omega^{i}\right\}\right)$ (similarly equipped with $k$ framed basepoints in $C_{X} \cap X^{\geq \mu_{3}}$ ) if:

- For each compact subset $K \subset X$ containing $\left\{\omega^{1}, \ldots, \omega^{k}\right\}$ there are smooth embeddings

converging $\mathcal{C}^{\infty}$ to an isometric embedding.

- For $i \neq j$ the distance $d_{N_{n}}\left(\omega_{n}^{i}, \omega_{n}^{j}\right)$ goes to infinity with $n$.

Let $\left(N_{n},\left\{\omega_{n}^{i}\right\}\right)$ be a sequence of hyperbolic 3-manifolds equipped with $k$ framed basepoints contained in the intersection $C_{N_{n}} \cap N^{\geq \mu_{3}}$. Assume the sequence $\left(N_{n},\left\{\omega_{n}^{i}\right\}\right)$ is converging geometrically to $\left(N,\left\{\omega^{i}\right\}\right)$. For any $r>0$ define

$$
K_{r}:=\bigcup_{i} B_{N}\left(\omega^{i}, r\right)
$$


Then geometric convergence provides a sequence of smooth embeddings

$$
\psi_{n}:\left(K_{r}, \omega^{1}, \omega^{2}, \ldots, \omega^{k}\right) \longrightarrow\left(N_{n}, \omega_{n}^{1}, \ldots, \omega_{n}^{k}\right)
$$

converging $\mathcal{C}^{\infty}$ to an isometric embedding.

Let us additionally assume that the convex cores $C_{N_{n}}$ are all homeomorphic, $C_{N_{n}}$ has incompressible boundary, and there is a uniform upper bound on the volume of $C_{N_{n}}$. Then by Proposition 4.2, for any $\delta>0$ there is an index $n_{\delta}$ such that $n>n_{\delta}$ implies:

- For any $x \in C_{N} \cap K_{r}, \psi_{n}(x)$ lies within distance $\delta$ from $C_{N_{n}}$.

- For any $y \in C_{N_{n}} \cap\left(\cup_{i} B_{N_{n}}\left(\omega_{n}^{i}, r\right)\right)$, y lies within distance $\delta$ from a point in the image $\psi_{n}\left(C_{N} \cap K_{r}\right)$.

In particular, it follows that

$$
\operatorname{Vol}\left(C_{N_{n}} \bigcap\left(\cup_{i} B_{N_{n}}\left(\omega_{n}^{i}, r\right)\right)\right) \longrightarrow \operatorname{Vol}\left(C_{N} \cap K_{r}\right),
$$

implying that

$$
\liminf \operatorname{Vol}\left(C_{N_{n}}\right) \geq \operatorname{Vol}\left(C_{N}\right) .
$$

For later reference let us record this as

Corollary 4.4. Let $\left(N_{n},\left\{\omega_{n}^{i}\right\}\right)$ be a sequence of hyperbolic 3-manifolds equipped with $k$ framed basepoints contained in the intersection $C_{N_{n}} \cap N \geq \mu_{3}$. Assume the sequence $\left(N_{n},\left\{\omega_{n}^{i}\right\}\right)$ is converging geometrically to $\left(N,\left\{\omega^{i}\right\}\right)$, the convex cores $C_{N_{n}}$ are all homeomorphic, $C_{N_{n}}$ has incompressible boundary, and there is a uniform upper bound on the volume of $C_{N_{n}}$. Then

$$
\liminf \operatorname{Vol}\left(C_{N_{n}}\right) \geq \operatorname{Vol}\left(C_{N}\right) .
$$

\section{Flattening the Bending Lamination}

Recall that $(M, P)$ is a connected pared 3-manifold with pared incompressible boundary. To avoid trivial cases, we assume $\pi_{1}(M)$ is not virtually abelian. The goal of this section is to prove the following theorem. (For definitions see Section 2.4.)

Theorem 5.1. Let $\lambda$ be the bending measured lamination on $\partial M-P$ of the boundary of the convex core of a minimally parabolic geometrically finite hyperbolic manifold $N \in \mathcal{I}(M, P)$ homeomorphic to the interior of $M$. Then for $0<\varepsilon<1$ there exists a geometrically finite minimally parabolic hyperbolic manifold $N_{\varepsilon} \in \mathcal{I}(M, P)$ homeomorphic to the interior of $M$ with bending measured lamination $\varepsilon \lambda$ such that

$$
\operatorname{Vol}\left(C_{N_{\varepsilon}}\right) \longrightarrow \frac{1}{2} \operatorname{Simp} \operatorname{Vol}(D(M, P)) \quad \text { as } \quad \varepsilon \rightarrow 0 .
$$

The existence of the manifolds $N_{\varepsilon}$ above follows quickly from the work of BonahonOtal [7] when $P=\emptyset$, and Lecuire 21] when $P \neq \emptyset$. (Note the assumption that $(M, P)$ has pared incompressible boundary does not imply that $M$ has incompressible boundary. Indeed, $(M, P)$ may be a handlebody pared along a multicurve which intersects every compressing disk. This is why it may be necessary to use results from [21.) The central point of Theorem [5.1 is ( $\dagger$ ). At first it may seem necessary to pass to a subsequence to obtain $(\dagger)$. However, we will quickly establish that the volumes $\operatorname{Vol}\left(C_{N_{\varepsilon}}\right)$ are monotonic in $\varepsilon$, allowing us to pass to subsequences freely in the proof.

Intuitively, the manifolds $N_{\varepsilon}$ should converge in some sense to a (possibly disconnected) manifold with Fuchsian ends, because all the geometric limits of the 
components of $\partial C_{N_{\varepsilon}}$ will be totally geodesic surfaces. Using a fair amount of Kleinian theory, the following argument makes this idea precise, and yields sufficient convergence to establish the limit $(\dagger)$.

The rest of the section is a proof of Theorem [5.1. Pick a measured geodesic lamination $\lambda$ on $\partial M-P$ realized by a minimally parabolic geometrically finite hyperbolic manifold $N_{1}$ equipped with a homeomorphism $\phi_{1}: M-P \longrightarrow C_{N_{1}}$.

By Theorem 2.2, for $0<\varepsilon<1$ there exists a geometrically finite hyperbolic manifold $N_{\varepsilon}$ such that there is a homeomorphism $\phi_{\varepsilon}: M-P \longrightarrow C_{N_{\varepsilon}}$, and the bending lamination on $\partial C_{N_{\varepsilon}}$ pulls back via $\phi_{\varepsilon}$ to $\varepsilon \lambda$ on $\partial M-P$. Moreover, the volumes $\operatorname{Vol}\left(C_{N_{\varepsilon}}\right)$ are non-increasing as $\varepsilon \rightarrow 0$. In particular, by Corollary 4.4 any geometric limit of the manifolds $N_{\varepsilon}$ (in the sense of Definition 4.3) is geometrically finite. This monotonicity also means that in proving Theorem 5.1 we are free to pass to subsequences.

We now apply a key compactness result due to Canary-Minsky-Taylor [13, Thm.5.5]. Let $\rho_{\varepsilon}: \pi_{1}(M) \longrightarrow \mathrm{PSl}_{2} \mathbb{C}$ be a representation given by $\left(N_{\varepsilon}, \phi_{\varepsilon}\right)\left(\rho_{\varepsilon}\right.$ is unique up to conjugation in $\mathrm{PSl}_{2} \mathbb{C}$ ). In the current notation, their theorem implies the following:

Theorem 5.2. [13, Thm.5.5] There exists a sequence $\varepsilon_{n} \rightarrow 0$, a sequence of homeomorphic re-markings $r_{n}:(M, P) \longrightarrow(M, P)$ which are the identity on the complement of the window of $(M, P)$, a collection $x$ of disjoint, non-parallel, homotopically non-trivial simple closed curves in the window base such that:

(1) If $M^{\prime}$ is a component of $M-X$ whose closure is not a thickened torus (where $X$ is the total space of the interval bundle over $x$ ), then there is a sequence of inner-automorphisms $\sigma_{n}$ of $\mathrm{PSl}_{2} \mathbb{C}$ such that the sequence of representations

$$
\left\{\left.\left(\sigma_{n} \circ \rho_{n} \circ r_{n *}\right)\right|_{\pi_{1}\left(M^{\prime}\right)}\right\}
$$

converges pointwise.

(2) If $c$ is a curve in $x$ which is not freely homotopic into $P$, then the length of the closed geodesic in $N_{\varepsilon_{n}}$ freely homotopic to $\left(\phi_{n} \circ r_{n}\right)(c)$ converges to zero.

For simplicity, let $N_{n}:=N_{\varepsilon_{n}}, \phi_{n}:=\phi_{\varepsilon_{n}}$, and $m_{n}:=\phi_{n} \circ r_{n}$.

Let $(W, R) \subset M$ be the union of the connected components of the characteristic submanifold of $(M, P)$ which are interval bundles over a surface with a non-abelian fundamental group. Recall that $\left(M_{\mathrm{acyl}}, Q\right)$ is the union of the pared acylindrical connected components of the complement of the characteristic submanifold of $(M, P)$. It will be convenient to move the components of $X$ into a standard form, and possibly make $X$ larger without violating the statement of Theorem 5.2 First, if a component of $X$ is isotopic rel $\partial M-P$ into $R \cup Q$, then isotope that component into $R \cup Q$. (This corresponds to having a component of $x$ which is isotopic into the boundary of the window base. We want to push it to the boundary of the window base.) Next, if $A$ is any component of $R \cup Q, A$ is not contained in $X$, and $A$ is freely homotopic in $M$ into $X$, then enlarge $X$ by adding the essential annulus $A$. Finally, if $A$ is any component of $R \cup Q, A$ is not contained in $X$, and $A$ is freely homotopic in $M$ into $P$, then enlarge $X$ by adding the essential annulus $A$. The statement of Theorem 5.2 remains true for the enlarged interval bundle $X$.

5.1. The interval bundle case. Pick a nonelementary component $M^{\prime}$ of the 3manifold $M-X$. Let $\widetilde{N}_{n}$ be the isometric covering of $N_{n}$ corresponding to the subgroup $m_{n *}\left(\pi_{1}\left(M^{\prime}\right)\right)<\pi_{1}\left(N_{n}\right)$. Let $\widetilde{m}_{n}: M^{\prime} \longrightarrow \widetilde{N}_{n}$ denote a resulting homotopy equivalence making the diagram 
commute up to homotopy.

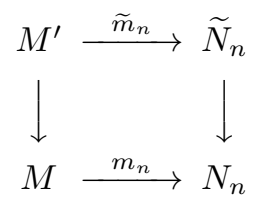

By conclusion (1) of Theorem 5.2 , the sequence $\left(\widetilde{N_{n}}, \widetilde{m_{n}}\right)$ converges in $\mathrm{AH}\left(M^{\prime}\right)$. Let $(\tilde{N}, \tilde{m}) \in \mathrm{AH}\left(M^{\prime}\right)$ be the algebraic limit. To be explicit, pick a basepoint $* \in M^{\prime} \subset M$, pick a framed basepoint $\widetilde{\omega}\left(\right.$ resp. $\widetilde{\omega}_{n}$ ) for $\widetilde{N}$ (resp. $\widetilde{N}_{n}$ ), and homotopically alter the markings $m_{n}, \widetilde{m}_{n}$, and $\widetilde{m}$ to make them maps of pointed spaces. By algebraic convergence, these choices can be made such that the holonomy representations

$$
\pi_{1}\left(M^{\prime}, *\right) \stackrel{\widetilde{m}_{n}}{\longrightarrow} \pi_{1}\left(\widetilde{N}_{n}, \widetilde{\omega}_{n}\right) \stackrel{\text { hol }}{\longrightarrow} \mathrm{PSl}_{2} \mathbb{C}
$$

converge pointwise to the holonomy representation

$$
\pi_{1}\left(M^{\prime}, *\right) \stackrel{\widetilde{m}}{\longrightarrow} \pi_{1}(\widetilde{N}, \widetilde{\omega}) \stackrel{\text { hol }_{\text {. }}}{\longrightarrow} \mathrm{PSl}_{2} \mathbb{C} .
$$

(The choice of a framed basepoint specifies the holonomy representation uniquely. See Section 2.3.) After possibly passing to a subsequence, we may assume that all framed basepoints are contained both in the convex core and in the $\mu_{3}$-thick part. Finally, let $\iota_{n}: \widetilde{N}_{n} \longrightarrow N_{n}$ denote the isometric covering map, and define the framed basepoint $\omega_{n}:=\iota_{n *} \widetilde{\omega}_{n}$.

Proposition 5.3. Suppose $(M, P)$ has an essential annulus or Möbius band which is contained in $M^{\prime}$ and is not freely homotopic into $X \cup P$. Then the sequence of framed manifolds $\left(N_{n}, \omega_{n}\right)$ converges geometrically to the algebraic limit $(\widetilde{N}, \widetilde{\omega})$, and $\tilde{N}$ is either a Fuchsian or an extended Fuchsian group.

Before proving this lemma, we state two corollaries.

Corollary 5.4. Suppose $M^{\prime}$ essentially intersects $W \subseteq M$. Then $M^{\prime} \subseteq W$.

The proof of Corollary 5.4 is immediate.

Corollary 5.5. Suppose $M^{\prime}$ essentially intersects $M_{\text {acyl }} \subseteq M$. Then $M^{\prime}$ is a component of $M_{a c y l}$.

Proof of Cor 5.5: Suppose $M^{\prime}$ essentially intersects $M_{\text {acyl }}$. Because $\left(M_{\text {acyl }}, Q\right)$ is pared acylindrical, it follows that a component $(L, \Pi)$ of $\left(M_{\mathrm{acyl}}, Q\right)$ is contained in $M^{\prime}$.

Pick an essential annulus $A$ on the frontier of $L$ as a submanifold of $M^{\prime}$. In particular, such an $A$ is not freely homotopic into $X$. If no such $A$ exists then $M^{\prime}=L$ and we are done. If $A$ is freely homotopic (in $M$ ) into $P$, then by our definition of $X, A$ is a component of $X$. Therefore $A$ is not freely homotopic into $X \cup P$. We may apply Proposition 5.3 to conclude that $(L, \Pi)$ is homotopic into the characteristic submanifold of $(M, P)$. This is a contradiction. Therefore there is no such essential annulus $A$, implying $M^{\prime}=L$.

Proof of Prop 5.3: The proof begins with a separate claim. Let $\mathbf{b l}\left(N_{n}\right)$ indicate the bending lamination on the boundary of the convex core of $N_{n}$. Recall that by definition $\mathbf{b l}\left(N_{n}\right)=\phi_{n *}\left(\varepsilon_{n} \lambda\right)$. 
Claim: For each $n$ there is an essential annulus or Möbius band $B_{n}$ of $C_{N_{n}}$ such that $m_{n}^{-1}\left(B_{n}\right) \subset(M, P)$ essentially intersects $M^{\prime} \subset M$. Moreover, the intersection number $i\left(\partial B_{n}, \mathbf{b l}\left(N_{n}\right)\right)$ goes to zero as $n$ goes to infinity.

(The annulus $m_{n}^{-1}\left(B_{n}\right) \subset M$ may change to follow the re-markings $r_{n}$.)

Proof of Claim: Let $\left\{\Sigma_{\ell}\right\}_{\ell}$ be a finite collection of essential annuli in the window of $(M, P)$ which are not freely homotopic into $P$, such that the window minus $\cup_{\ell} \Sigma_{\ell}$ is a collection of topological balls and solid tori, and each component of the window not freely homotopic into $P$ contains a component of the collection $\left\{\Sigma_{\ell}\right\}_{\ell}$. By hypothesis the collection $\left\{\Sigma_{\ell}\right\}_{\ell}$ is not empty. For each pared homeomorphism $r_{n}:(M, P) \longrightarrow(M, P)$, the submanifold $M^{\prime}$ will essentially intersect $r_{n}^{-1}\left(\Sigma_{\ell_{n}}\right) \subset$ $(M, P)$ for some $\ell_{n}$.

Let $B_{n} \subset C_{N_{n}}$ be the essential annulus or Möbius band $\phi_{n}\left(\Sigma_{\ell_{n}}\right) \subset N_{n}$. The intersection number

$$
i\left(\partial B_{n}, \mathbf{b l}\left(N_{n}\right)\right)=i\left(\phi_{n}^{-1}\left(\partial B_{n}\right), \varepsilon_{n} \lambda\right)=\varepsilon_{n} \cdot i\left(\Sigma_{\ell_{n}}, \lambda\right) \leq \varepsilon_{n} \cdot \max _{\ell}\left\{i\left(\Sigma_{\ell}, \lambda\right)\right\}
$$

goes to zero. Also, $m_{n}^{-1}\left(B_{n}\right)=r_{n}^{-1}\left(\Sigma_{\ell_{n}}\right)$ essentially intersects $M^{\prime}$. This proves the claim.

Let $\gamma_{n}^{*}$ be the closed geodesic freely homotopic into $B_{n} . B_{n} \subset C_{N_{n}}$ is homotopic rel boundary to an essential annulus or Möbius band $B_{n}^{*}$ containing $\gamma_{n}^{*}$, such that $\partial B_{n}^{*} \subset \partial C_{N_{n}}$ is a (possibly disconnected) closed geodesic in the intrinsic hyperbolic metric on $\partial C_{N_{n}}$. By the claim, $\partial B_{n}^{*}$ is very lightly bent, meaning that $i\left(\partial B_{n}^{*}, \mathbf{b f}\left(N_{n}\right)\right)$ is small. After adjusting $B_{n}^{*}$ by an isotopy fixing both $\partial B_{n}^{*}$ and $\gamma_{n}^{*}$, we may assume that the supremum

$$
\sup _{y \in B_{n}^{*}} d_{N_{n}}\left(y, \gamma_{n}^{*}\right)
$$

is attained at a point in $\partial B_{n}^{*}$. (Simply straightening the annulus rel $\partial B_{n}^{*}$ and $\gamma_{n}^{*}$ would suffice.)

Let $\left\{g_{n}^{1}, \ldots, g_{n}^{k}\right\}$ be a generating set for $\pi_{1}\left(\widetilde{N}_{n}, \widetilde{\omega}_{n}\right)$. Let $g_{n}^{i *}$ be the based geodesic in $N_{n}$ corresponding to $g_{n}^{i}$. We chose $B_{n}$ so that $m_{n}^{-1}\left(B_{n}\right) \subset M$ would intersect $M^{\prime}$ essentially. Therefore one of the based geodesics $g_{n}^{i *}$ of the generating set must intersect $B_{n}^{*}$. By algebraic convergence, the lengths of the based geodesics $\left\{g_{n}^{i *}\right\}_{n, i}$ have a finite supremum.

We now show that the length of $\gamma_{n}^{*}$ has a uniform lower bound. Suppose that up to extracting a subsequence the length of $\gamma_{n}^{*}$ is going to zero. Since $\partial B_{n}^{*}$ is very lightly bent, Lemma 2.5 implies that the length of $\partial B_{n}^{*}$ is also going to zero. This forces the entire surface $B_{n}^{*} \subset N_{n}$ to be contained deep inside the Margulis tube corresponding to $\gamma_{n}^{*}$. In particular, this produces a sequence of based geodesics $g_{n}^{i_{n} *}$ with unbounded length, contradicting algebraic convergence. Therefore the length of $\gamma_{n}^{*}$ has a uniform lower bound.

Once we know that $\gamma_{n}^{*}$ is uniformly long, the inequality of Lemma 2.5 becomes stronger, implying that $\partial B_{n}^{*}$ lies inside a $d_{n}$-neighborhood of $\gamma_{n}^{*}$, where $d_{n} \rightarrow 0$. By the construction of $B_{n}^{*}$ this forces all of $B_{n}^{*}$ into a $d_{n}$-neighborhood of $\gamma_{n}^{*}$. Since the based geodesic $g_{n}^{i_{n} *}$ intersects $B_{n}^{*}$, it follows that $\gamma_{n}^{*} \subset N_{n}$ passes within a uniformly bounded distance from the basepoint $\omega_{n}$ of $N_{n}$.

In the universal cover $\mathbb{H}^{3}$ with basepoint $o \in \mathbb{H}^{3}$, we can lift $\gamma_{n}^{*}$ to a geodesic passing within a uniformly bounded distance from $o$. This specifies a lift of the entire surface $B_{n}^{*}$ to an infinite closed annulus. Choose a point on each component 
of this infinite closed annulus, call them $y_{n}^{1}$ and $y_{n}^{2}$. At $y_{n}^{i}$ pick a hyperbolic plane $\mathfrak{h}_{n}^{i}$ intersecting the convex hull of $\pi_{1}\left(N_{n}, \omega_{n}\right) \subset \mathbb{H}^{3}$ only at the point $y^{i}$ (this notation is defined in Section 2.3). Such a plane is called a support plane at $y^{i}$. We chose $y_{n}^{1}$ and $y_{n}^{2}$ to lie on different components of the convex hull of $\pi_{1}\left(N_{n}, \omega_{n}\right)$. This forces the planes $\mathfrak{h}_{n}^{1}$ and $\mathfrak{h}_{n}^{2}$ to be disjoint. Moreover, they bound a closed region of $\mathbb{H}^{3}$ containing the convex hull of $\pi_{1}\left(\widetilde{N}_{n}, \widetilde{\omega}_{n}\right)$ and the convex hull of $\pi_{1}\left(N_{n}, \omega_{n}\right)$. By choosing the points $y_{n}^{1}$ and $y_{n}^{2}$ to be as near to $o$ as possible, we can guarantee there is a geodesic segment $\sigma_{n}$ of length less than $4 d_{n}$ from $\mathfrak{h}_{n}^{1}$ to $\mathfrak{h}_{n}^{2}$.

Since the geodesic $\gamma_{n}^{*}$ passes within a uniformly bounded distance from the basepoint $\omega^{1}$ of $N_{n}$, the geodesic segment $\sigma_{n}$ stretching between the support planes $\mathfrak{h}_{n}^{1}$ and $\mathfrak{h}_{n}^{2}$ can be chosen to stay within a uniformly bounded distance from the basepoint $o \in \mathbb{H}^{3}$. Since the length of $\sigma_{n}$ is going to zero, the support planes $\mathfrak{h}_{n}^{1}$ and $\mathfrak{h}_{n}^{2}$ are converging to each other. Therefore after passsing to a subsequence we can find a round circle at infinity $\mathbb{S}^{1} \subset \partial \mathbb{H}^{3}$ such that: for $k=1,2$ the round circles $\partial \mathfrak{h}_{n}^{k} \subset \partial \mathbb{H}^{3}$ are converging in the Hausdorff topology to $\mathbb{S}^{1}$. Therefore the limit sets of the groups $\pi_{1}\left(N_{n}, \omega_{n}\right)$ and $\pi_{1}\left(\widetilde{N}_{n}, \widetilde{\omega}_{n}\right)$ are contained in smaller and smaller neighborhoods of $\mathbb{S}^{1} \subset \partial \mathbb{H}^{3}$.

Suppose that the fundamental group of the algebraic limit $\pi_{1}(\widetilde{N}, \widetilde{\omega})<\mathrm{PSl}_{2} \mathbb{C}$ contains an element $\gamma$ which does not preserve $\mathbb{S}^{1} \subset \partial \mathbb{H}^{3}$. Let $U \subset \partial \mathbb{H}^{3}$ be an open neighborhood containing $\mathbb{S}^{1}$ such that $\gamma \cdot \theta \notin \bar{U}$ for some $\theta \in \mathbb{S}^{1}$. We can find a small neighborhood $V \subset U$ of $\theta$ such that for sufficiently large $n$, the group $\pi_{1}\left(\widetilde{N}_{n}, \widetilde{\omega}_{n}\right)$ contains an element $\gamma_{n}$ satisfying $\gamma_{n} . V \cap U=\emptyset$. Moreover, for sufficiently large $n$ the limit set of $\pi_{1}\left(N_{n}, \omega_{n}\right)$ is contained in $U$ and intersects $V$ in at least one point $p$. (This point $p$ exists because $(M, P)$ has pared incompressible boundary, forcing each component of the boundary of the convex hull of $\pi_{1}\left(N_{n}, \omega_{n}\right)$ to be a pleated hyperbolic plane. At least one of these pleated hyperbolic planes limits onto a Jordan curve in $\partial \mathbb{H}^{3}$ which is homotopically nontrivial in the annulus $\overline{\operatorname{Hull}\left(\mathfrak{h}_{n}^{1} \cup \mathfrak{h}_{n}^{2}\right)} \cap \partial \mathbb{H}^{3}$. This annulus is converging in the Hausdorff topology to $\mathbb{S}^{1}$.) Since the action of $\pi_{1}\left(N_{n}, \omega_{n}\right)$ on $\partial \mathbb{H}^{3}$ preserves its own limit set, this is a contradiction. Therefore the fundamental group of the algebraic limit $\pi_{1}(\widetilde{N}, \widetilde{\omega})$ preserves $\mathbb{S}^{1} \subset \partial \mathbb{H}^{3}$.

Let $\mathbb{H}^{2} \subset \mathbb{H}^{3}$ be the hyperbolic plane spanning $\mathbb{S}^{1}$. To see that the algebraic limit $\pi_{1}(\widetilde{N}, \widetilde{\omega})$ acts on $\mathbb{H}^{2}$ with finite co-area, consider any component $(S, \partial S)$ of $\overline{\partial M-P} \cap \overline{M^{\prime}}$. Each component of $\partial S$ is mapped to curves of smaller and smaller length (by conclusion (2) of Theorem [5.2). So in the algebraic limit the surface $(S, \partial S)$ must correspond to a quasi-Fuchsian subgroup of $\pi_{1}(\widetilde{N}, \widetilde{\omega})$. Since the full algebraic limit $(\widetilde{N}, \widetilde{\omega})$ preserves $\mathbb{S}^{1}$, and it contains a quasi-Fuchsian subgroup, it must be either Fuchsian or extended Fuchsian (with finite co-area).

After possibly passing to a subsequence, let $(Z, \tau)$ be the geometric limit of the framed manifolds $\left(N_{n}, \omega_{n}\right)$. By the previous paragraph and Proposition 4.1 the Kleinian group $\pi_{1}(Z, \tau)<\mathrm{PSl}_{2} \mathbb{C}$ corresponding to $(Z, \tau)$ is geometrically finite and preserves the hyperbolic plane $\mathbb{H}^{2} \subset \mathbb{H}^{3}$ spanning $\mathbb{S}^{1}$. Since the algebraic limit $\widetilde{N}$ is geometrically finite also, by considering convex core area one sees that $(\widetilde{N}, \widetilde{\omega})$ is a finite cover of the geometric limit $(Z, \tau)$. The next paragraph will use this to show that the covering map is a homeomorphism.

The locally isometric covering map $(\widetilde{N}, \widetilde{\omega}) \longrightarrow(Z, \tau)$ induces an inclusion

$$
\pi_{1}(\widetilde{N}, \widetilde{\omega}) \leq \pi_{1}(Z, \tau)<\mathrm{PSl}_{2} \mathbb{C}
$$


Suppose there is an element $g \in \pi_{1}(Z, \tau)-\pi_{1}(\widetilde{N}, \widetilde{\omega})$. Since the covering map has finite degree, there is a $k \in \mathbb{N}$ such that $g^{k} \in \pi_{1}(\widetilde{N}, \widetilde{\omega})$. Therefore there exists a sequence of natural numbers $\left\{n_{i}\right\}$, a sequence $\alpha_{n_{i}}$ in $\pi_{1}(M, *)$, and a $\beta \in \pi_{1}\left(M^{\prime}, *\right)$ such that

$$
g=\lim _{i} m_{n_{i} *}\left(\alpha_{n_{i}}\right), \quad \text { and } \quad g^{k}=\lim _{i} \widetilde{m}_{n_{i} *}(\beta),
$$

where

$$
m_{n_{i} *}: \pi_{1}(M, *) \longrightarrow \pi_{1}\left(N_{n}, \omega_{n}\right) \quad \text { and } \quad \widetilde{m}_{n_{i} *}: \pi_{1}\left(M^{\prime}, *\right) \longrightarrow \pi_{1}\left(\widetilde{N}_{n}, \widetilde{\omega}_{n}\right)
$$

are the maps on fundamental groups induced by the markings. This implies that $m_{n_{i} *}\left(\alpha_{n_{i}}^{k} \beta^{-1}\right)$ converges to the identity matrix as $i \rightarrow \infty$. The algebraic convergence of the representations $\widetilde{m}_{n *} \rightarrow \widetilde{m}$ forces the existence of an $\eta>0$ such that every basepoint $\omega_{n} \in N_{n}$ is contained in the $\eta$-thick part of $N_{n}$. (Failure would force every loxodromic element of $\pi_{1}\left(\widetilde{N}_{n}, \widetilde{\omega}_{n}\right)<\pi_{1}\left(N_{n}, \omega_{n}\right)$ to translate the base point $\widetilde{\omega}_{n}$ arbitrarily far for $n \gg 0$, violating algebraic convergence.) This uniform thickness $\eta$ yields a uniform neighborhood $U \subset \mathrm{PSl}_{2} \mathbb{C}$ of the identity such that

$$
\pi_{1}\left(N_{n}, \omega_{n}\right) \cap U=\text { identity for all } n .
$$

Therefore for $i \gg 0, \alpha_{n_{i}}^{k}=\beta \in \pi_{1}(M, *)$. In the fundamental group of a hyperbolic 3 -manifold, group elements with at least one $k^{\text {th }}$ root have a unique $k^{\text {th }}$ root. So there is an element $\gamma \in \pi_{1}(M, *)$ satisfying $\gamma^{k}=\beta \in \pi_{1}\left(M^{\prime}, *\right)$, and $\alpha_{n_{i}}=\gamma$ for all $i \gg 0$. It follows that in fact $\gamma \in \pi_{1}\left(M^{\prime}, *\right)$. So finally we have shown that $g=\lim _{i} m_{n_{i} *}(\gamma)=\lim _{i} \widetilde{m}_{n_{i} *}(\gamma)$, implying that $g$ is an element of the algebraic limit $\pi_{1}(\widetilde{N}, \widetilde{\omega})$. (It is a standard fact from the theory Kleinian groups that if the algebraic limit is a finite index subgroup of the geometric limit, then they are the same. See, for example, the proof of [19, Prop.4.2]. The above argument is an adaptation of the proof of this fact to the current setting.)

Thus we have shown that $\pi_{1}(Z, \tau)=\pi_{1}(\widetilde{N}, \widetilde{\omega})<\mathrm{PSl}_{2} \mathbb{C}$.

Using Corollaries 5.4 and 5.5 we may conclude that:

Each non-elementary component $M^{\prime}$ of $M-X$ is either a sub-interval bundle of $(W, R)$, or is equal to a component of $\left(M_{a c y l}, Q\right)$. Conversely, each component of $\left(M_{\text {acyl }}, Q\right)$ is a component of $M-X$.

5.2. The pared acylindrical case. Pick a component $(L, \Pi)$ from the pared acylindrical manifold $\left(M_{\text {acyl }}, Q\right)$. To avoid too much clutter, we will now re-use some previous notation. We now let $\widetilde{N}_{n}$ denote an isometric covering of $N_{n}$ corresponding to the conjugacy class of the subgroup $m_{n *}\left(\pi_{1}(L)\right)<\pi_{1}\left(N_{n}\right)$. Let $\widetilde{m}_{n}: L \longrightarrow \widetilde{N}_{n}$ denote a resulting homotopy equivalence making the diagram

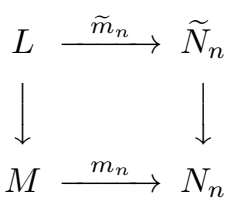

commute up to homotopy. By Theorem $\left[5.2\right.$, the sequence $\left\{\left(\widetilde{N}_{n}, \widetilde{m}_{n}\right)\right\} \subset \mathrm{H}(L)$ converges algebraically to a manifold $(\widetilde{N}, \widetilde{m}) \in \mathrm{H}(L, \Pi)$.

Pick a basepoint $* \in L \subset M$, pick a framed basepoint $\widetilde{\omega}$ (resp. $\widetilde{\omega}_{n}$ ) for $\widetilde{N}$ (resp. $\widetilde{N}_{n}$ ), and homotopically alter the markings $m_{n}, \widetilde{m}_{n}$, and $\widetilde{m}$ to make them maps of 
pointed spaces. By algebraic convergence, these choices can be made such that the holonomy representations

$$
\pi_{1}(L, *) \stackrel{\widetilde{m}_{n}}{\longrightarrow} \pi_{1}\left(\widetilde{N}_{n}, \widetilde{\omega}_{n}\right) \stackrel{\text { hol }_{1}}{\longrightarrow} \mathrm{PSl}_{2} \mathbb{C}
$$

converge pointwise to the holonomy representation

$$
\pi_{1}(L, *) \stackrel{\widetilde{m}}{\longrightarrow} \pi_{1}(\widetilde{N}, \widetilde{\omega}) \stackrel{\text { hol }_{1}}{\longrightarrow} \mathrm{PSl}_{2} \mathbb{C} .
$$

Up to passing to a subsequence, we may assume that all framed basepoints are contained both in the convex core and in the $\mu_{3}$-thick part. Finally, let $\iota_{n}: \widetilde{N}_{n} \longrightarrow$ $N_{n}$ denote the isometric covering map, and define the framed basepoint $\omega_{n}:=$ $\iota_{n *} \widetilde{\omega}_{n}$.

After possibly passing to a subsequence, let $(Z, \tau)$ be a geometric limit of a subsequence of $\left\{\left(N_{n}, \omega_{n}\right)\right\}$. By Proposition 4.4 $\operatorname{Vol}\left(C_{Z}\right) \leq \liminf \operatorname{Vol}\left(C_{\widetilde{N}_{n}}\right)<\infty$. Therefore $(Z, \tau)$ is geometrically finite. Since there is a locally isometric covering map $(\widetilde{N}, \widetilde{\omega}) \rightarrow(Z, \tau)$, a standard covering theorem due to Thurston now implies that the algebraic limit $\widetilde{N}$ is also geometrically finite [12, Thm.2.1].

Lemma 5.6. The algebraic limit of the sequence $\left(\widetilde{N}_{n}, \widetilde{m}_{n}\right) \in H(L)$ is minimally parabolic in the set $H(L, \Pi)$.

Proof: $\quad(L, \Pi)$ is pared acylindrical. For some $\zeta<\mu_{3}$ we have the homotopy equivalence $\widetilde{m}: L \longrightarrow C_{\widetilde{N}} \cap(\widetilde{N})^{\geq \zeta}$ given by the marking. Using conclusion (2) of Theorem 5.2, we know that (up to homotopy) $\widetilde{m}$ takes $\Pi$ into $C_{\widetilde{N}} \cap \partial(\widetilde{N})^{\geq \zeta}$. Using a topological rigidity theorem due to Johannson, we can conclude that $\widetilde{m}$ is homotopic to a homeomorphism taking $\Pi$ into $C_{\widetilde{N}} \cap \partial(\widetilde{N}) \geq \zeta$ [18, Lem.X.23,pg.235]. Let $\widetilde{m}$ now denote this homeomorphism.

Suppose $(\widetilde{N}, \widetilde{m})$ is not minimally parabolic in $\mathrm{H}(L, \Pi)$. Then there is an essential curve $c_{1} \subset L$ not freely homotopic into $\Pi$, such that $c_{1}$ is mapped by $\widetilde{m}$ to a parabolic curve in $\widetilde{N}$. Since $\widetilde{m}$ is a homeomorphism, the curve $c_{1}$ is freely homotopic into $\partial L-\Pi$.

Therefore we may assume there exists an essential curve $c_{1} \subset \partial L-\Pi$ such that the length of the closed geodesic $\widetilde{m}_{n}\left(c_{1}\right)^{*}$ freely homotopic to $\widetilde{m}_{n}\left(c_{1}\right) \subset \widetilde{N}_{n}$ is going to zero. Pick an essential curve $c_{2} \subset \partial L-\Pi$ which intersects $c_{1}$ essentially in $\partial L-\Pi$. Since the re-markings $r_{n}:(M, P) \longrightarrow(M, P)$ are the identity on the pared acylindrical component $(L, \Pi)$, we may apply Lemma 2.5 to conclude that (for $i=1,2) \tilde{m}_{n}\left(c_{i}\right)$ is homotopic to a curve in $\partial C_{\widetilde{N}_{n}}$ whose length is uniformly close (i.e. with small multiplicative and small additive error) to the length of the closed geodesic $\widetilde{m}_{n}\left(c_{i}\right)^{*}$ homotopic to $\widetilde{m}_{n}\left(c_{i}\right)$. Therefore $\widetilde{m}_{n}\left(c_{1}\right)$ is homotopic to a very short curve in $\partial C_{\widetilde{N}_{n}}$, implying that every curve in $\partial C_{\widetilde{N}_{n}}$ homotopic to $\widetilde{m}_{n}\left(c_{2}\right)$ is very long. Therefore $\widetilde{m}_{n}\left(c_{2}\right)^{*}$ is very long, with length going to infinity. This contradicts algebraic convergence. Therefore there exists no such curve $c_{1} \subset \partial L$, and we have proven that the algebraic limit of the sequence $\left(\widetilde{N}_{n}, \widetilde{m}_{n}\right) \in \mathrm{H}(L)$ is minimally parabolic in the set $\mathrm{H}(L, \Pi)$.

Lemma 5.7. There is a sequence $a_{n} \rightarrow \infty$ with the following property: if $c \subset N_{n}$ is a homotopically nontrivial closed path such that $m_{n}^{-1}(c) \subset M$ essentially intersects $L \subset M$, and is not freely homotopic into $L$, then the length of $c \subset N_{n}$ is at least $a_{n}$. 
Proof: $\quad$ Pick an essential annulus $A \subset M$ on the frontier of $L \subset M$. By previous considerations, $A$ must be a component of $X$. As a simplifying assumption, let us assume that $A$ is not freely homotopic into $P$. The proof in the general case requires only slight modifications, and is left to the reader. Let $\left(A_{n}^{*}, \partial A_{n}^{*}\right) \subset\left(C_{N_{n}}, \partial C_{N_{n}}\right)$ be an embedded essential annulus freely homotopic to $m_{n}(A) \subset N_{n}$ such that $A_{n}^{*}$ contains the closed geodesic $\gamma_{n}^{*} \subset C_{N_{n}}$ freely homotopic into $m_{n}(A)$, and $\partial A_{n}^{*} \subset$ $\partial C_{N_{n}}$ is a pair of closed geodesics in the intrinsic metric on $\partial C_{N_{n}}$. By pulling tight a homotopy from $\partial A_{n}^{*}$ to $\gamma_{n}^{*}$ we may assume that $A_{n}^{*}$ is foliated by curves of length less than the length of $\partial A_{n}^{*}$.

We claim that the length of $\partial A_{n}^{*}$ is going to zero. To see this, recall that the remarking homeomorphisms $r_{n}:(M, P) \longrightarrow(M, P)$ are the identity off the window of $(M, P)$. Therefore off the window, the support of the bending lamination $\mathbf{b l}\left(N_{n}\right)$ is "fixed", i.e. the support of $\left(m_{n}^{-1}\right)_{*}\left(\mathbf{b l}\left(N_{n}\right)\right) \subset \partial M-P$ is constant off the window of $(M, P)$. Since the total mass of $\mathbf{b l}\left(N_{n}\right)$ is going to zero, we may again use Lemma 2.5 to conclude that the length of each component of $\partial A_{n}^{*}$ is close to the length of $\gamma_{n}^{*}$. By conclusion (2) of Theorem [5.2, the length of $\gamma_{n}^{*}$ is going to zero. This proves the claim.

For $n \gg 0$, let $T_{n} \subset N_{n}$ be the $\mu_{3}$-Margulis tube about the short closed geodesic $\gamma_{n}^{*} \subset N_{n}$. Since the length of $\partial A_{n}^{*}$ is going to zero, $A_{n}^{*}$ is foliated by very short curves. Therefore the length $a_{n}$ of the shortest path from $\partial T_{n}$ to $A_{n}^{*}$ goes to infinity.

Now let $c \subset N_{n}$ be a homotopically nontrivial path such that $m_{n}^{-1}(c) \subset M$ essentially intersects the annulus $A \subset M$. We claim that the length of $c$ is at least $a_{n}$. Without a loss of generality, we may assume that $c$ is a closed geodesic in $C_{N_{n}}$. Then by topological considerations $c$ cannot be contained in $T_{n}$, and it must intersect $A_{n}^{*}$.

Since the frontier of $L \subset M$ has only a finite number of components, performing the above procedure on each of them proves the lemma.

Recall that $(Z, \tau)$ is the geometric limit of the framed manifolds $\left(N_{n}, \omega_{n}\right)$, and $Z$ is geometrically finite by Corollary 4.4

Corollary 5.8. $(Z, \tau)$ is the geometric limit of the sequence of framed manifolds $\left(\widetilde{N}_{n}, \widetilde{\omega}_{n}\right)$.

Proof: After possibly passing to a subsequence, let $\left(Z^{\prime}, \tau^{\prime}\right)$ be the geometric limit of the sequence $\left(\widetilde{N}_{n}, \widetilde{\omega}_{n}\right)$. Then there is a locally isometric covering map $\left(Z^{\prime}, \tau^{\prime}\right) \rightarrow(Z, \tau)$. Suppose this map is not a homeomorphism. Then there is an element in $\pi_{1}(Z, \tau)$ which is not in the image of the induced homomorphism on fundamental groups. This element yields a sequence of elements in $\pi_{1}\left(N_{n}, \omega_{n}\right)$ which are not in the image of the homomorphism $\pi_{1}\left(\widetilde{N}_{n}, \widetilde{\omega}_{n}\right) \rightarrow \pi_{1}\left(N_{n}, \omega_{n}\right)$ induced by the covering map, and are represented by curves $\gamma_{n}$ based at $\omega_{n}$ of uniformly bounded length.

Pick an essential curve $b \subset L$ based at $*$ which is not freely homotopic out of $L$. Then the closed curve $c_{n}$ formed by concatenating $b$ and $m_{n}^{-1}\left(\gamma_{n}\right)$ essentially intersects $L$ and is not freely homotopic into $L$. Therefore the length of a closed curve in $N_{n}$ homotopic to $m_{n}\left(c_{n}\right)$ must have length at least $a_{n}$ (where $a_{n}$ is the constant of Lemma 5.7). Algebraic convergence provides curves based at $\omega_{n}$ of uniformly bounded length which are homotopic to $m_{n}(b)$. Since $a_{n} \rightarrow \infty$, this is a contradiction. 
Corollary 5.9. There is a sequence $\ell_{n} \rightarrow 0$ such that: if $A \subset(M, P)$ is an essential annulus on the frontier of $L$ and $\widetilde{m}_{n}(A) \subset \widetilde{N}_{n}$ is freely homotopic into a component $S$ of $\partial C_{\widetilde{N}_{n}}$ then $\widetilde{m}_{n}(A)$ is freely homotopic to a curve on $S$ of length less than $\ell_{n}$.

Note that because $(L, \Pi)$ is pared acylindrical, $\widetilde{m}_{n}(A)$ is freely homotopic into either two components of $\partial C_{\widetilde{N}_{n}}$ (when it is parabolic) or exactly one component of $\partial C_{\widetilde{N}_{n}}$ (when it is hyperbolic).

Proof: In the notation of the proof of Lemma 5.7, $\partial A_{n}^{*}$ has length going to zero and lies in $\partial C_{N_{n}}$. Lift $\partial A_{n}^{*}$ to $\widetilde{N}_{n}$, where the lifts lie in the complement $\widetilde{N}_{n}-\operatorname{int}\left(C_{\widetilde{N}_{n}}\right)$. Project the lifts down to $\partial C_{\widetilde{N}_{n}}$ to obtain the desired short curves.

Lemma 5.10. The sequence of framed manifolds $\left(N_{n}, \omega_{n}\right)$ converges geometrically to $(\widetilde{N}, \widetilde{\omega})$.

Proof: By Corollary [5.8, it suffices to prove that the sequence $\left(\widetilde{N}_{n}, \widetilde{\omega}_{n}\right)$ converges geometrically to its algebraic $\operatorname{limit}(\widetilde{N}, \widetilde{\omega})$. Recall that $(\widetilde{N}, \widetilde{m})$ is a geometrically finite minimally parabolic manifold in the deformation set $\mathrm{H}(L, \Pi)$.

The goal will be to apply a criterion for strong convergence due to JorgensenMarden [19, Sec.4.7] (see also [24, Thm.4.2]). Namely, for each cyclic subgroup $\langle a\rangle<\pi_{1}(L, *)$ corresponding to an annular component $A$ of $\Pi$, it suffices to show that the groups $\left\langle\widetilde{m}_{n *}(a)\right\rangle<\pi_{1}\left(\widetilde{N}_{n}, \widetilde{\omega}_{n}\right)$ converge geometrically to the rank one cusp $\left\langle\widetilde{m}_{*}(a)\right\rangle<\pi_{1}(\widetilde{N}, \widetilde{\omega})$. If for an infinite number of values $n$ the subgroup $\left\langle\widetilde{m}_{n *}(a)\right\rangle$ is a parabolic subgroup, then the conclusion follows from algebraic convergence. So let us assume without a loss of generality that $\widetilde{m}_{n *}(a)$ is a hyperbolic element of $\pi_{1}\left(\widetilde{N}_{n}, \widetilde{\omega}_{n}\right)$ for all $n$.

As a first step, we must show that a compressible curve on $\partial C_{\widetilde{N}_{n}}$ cannot be short. Let $\lambda_{n}$ be the minimum length of a closed geodesic on a hyperbolic surface which essentially intersects a geodesic of length $\ell_{n}$ (where $\ell_{n}$ is the sequence of constants from Corollary (5.9). Then $\lambda_{n} \rightarrow \infty$. Let $c \subset \partial C_{\widetilde{N}_{n}}$ be a closed curve which is essential on $\partial C_{\widetilde{N}_{n}}$ and homotopically trivial in $\widetilde{N}_{n}$. Since $(L, \Pi)$ is pared acylindrical, every representative of the free homotopy class $\widetilde{m}_{n}^{-1}(c) \subset L$ lying on $\partial L$ must intersect $\Pi \subset \partial L$. Applying Corollary [5.9, $c$ must intersect an essential curve of $\partial C_{\widetilde{N}_{n}}$ of length less than $\ell_{n}$. Therefore the length of $c$ must be at least $\lambda_{n}$. Since $\lambda_{n} \rightarrow \infty$, this completes the first step.

The isometry $a_{n}:=\widetilde{m}_{n *}(a)$ is hyperbolic, and $(L, \Pi)$ is pared acylindrical. It follows that $\widetilde{m}_{n}(A) \subset \widetilde{N}_{n}$ is freely homotopic into a unique component of $\partial C_{N_{n}}$. In fact, $\widetilde{m}_{n}(A)$ is freely homotopic to a short curve in $\partial C_{\widetilde{N}_{n}}$ (Corollary [5.9), and all the compressible curves in $\partial C_{\widetilde{N}_{n}}$ are long (the previous paragraph). We may therefore apply a theorem of Bridgeman-Canary [9, Thm.1'] to conclude that $a_{n}$ can be represented in the conformal boundary of $\widetilde{N}_{n}$ by a short curve, i.e. with length in the Poincaré metric going to 0 .

We can now finish the proof using the fairly complete description of geometric convergence (in the geometrically finite setting) given by Proposition 4.7 and Theorem 4.9 of [19. Assume the groups $\left\langle\widetilde{m}_{n *}(a)\right\rangle$ converge geometrically to a rank two cusp. Using the two aforementioned results, the curve $\widetilde{m}_{*}(a) \subset \widetilde{N}$ is represented in the conformal boundary of $\widetilde{N}$ by a geodesic, i.e. a curve with strictly positive length. This violates the conclusion of the previous paragraph together with the 
fact that the domains of discontinuity of the manifolds $\widetilde{N}_{n}$ converge in the sense of Caratheodory to the domain of discontinuity of $\widetilde{N}$. (This convergence is conclusion (i) of [19, Thm.4.9].) We must therefore have the desired strong convergence.

Thus by examining each nonelementary component of $M-X$ we have proven the following proposition for some $0<\eta_{0}<\mu_{3}$ :

Proposition 5.11. After passing to a subsequence there exists a sequence of sets of $k$ framed basepoints $\left\{\omega_{n}^{i}\right\}_{i=1}^{k} \subset C_{N_{n}} \cap N \geq \eta_{0}$, a (possibly disconnected) geometrically finite hyperbolic manifold $N$ with $k$ components, and a set of $k$ framed basepoints $\left\{\omega^{i}\right\}_{i=1}^{k} \subset C_{N} \cap N^{\geq \eta_{0}}$, such that $\left(N_{n},\left\{\omega_{n}^{i}\right\}\right)$ converges geometrically to $\left(N,\left\{\omega^{i}\right\}\right)$ in the sense of Definition 4.3 .

5.3. Concluding arguments. We now recall the notation of Corollary 4.4. For any $r>0$ define

$$
K_{r}:=\bigcup_{i} B_{N}\left(\omega^{i}, r\right)
$$

Then the geometric convergence of Proposition 5.11 provides a sequence of smooth embeddings

$$
\psi_{n}:\left(K_{r}, \omega^{1}, \omega^{2}, \ldots, \omega^{k}\right) \longrightarrow\left(N_{n}, \omega_{n}^{1}, \ldots, \omega_{n}^{k}\right)
$$

converging $\mathcal{C}^{\infty}$ to an isometric embedding. For any $\delta>0$ there is an index $n_{\delta}$ such that $n>n_{\delta}$ implies:

- For any $x \in C_{N} \cap K_{r}, \psi_{n}(x)$ lies within distance $\delta$ from $C_{N_{n}}$.

- For any $y \in C_{N_{n}} \cap\left(\cup_{i} B_{N_{n}}\left(\omega_{n}^{i}, r\right)\right)$, y lies within distance $\delta$ from a point in the image $\psi_{n}\left(C_{N} \cap K_{r}\right)$.

In particular, it follows that

$$
\operatorname{Vol}\left(C_{N_{n}} \bigcap\left(\cup_{i} B_{N_{n}}\left(\omega_{n}^{i}, r\right)\right)\right) \longrightarrow \operatorname{Vol}\left(C_{N} \cap K_{r}\right) .
$$

Lemma 5.12. Each component of $C_{N}$ is either a totally geodesic surface or a 3-manifold with totally geodesic boundary.

Proof: $\quad$ Pick an essential closed curve $c \subset \partial C_{N}$ which is a geodesic in the intrinsic hyperbolic metric on $\partial C_{N}$. Let $c^{*} \subset C_{N}$ be the closed geodesic freely homotopic to $c$. To prove the lemma it suffices to show that $c$ and $c^{*}$ have the same length. Using the almost isometric embeddings $\psi_{n}$ above, for each manifold $N_{n}$ we can find a geodesic $c_{n}^{*} \subset N_{n}$ homotopic to $\psi_{n}\left(c^{*}\right)$. Homotopic to $c_{n}^{*}$ there is a curve $c_{n} \subset \partial C_{N_{n}}$ which is a geodesic in the instrinsic metric on $\partial C_{N_{n}}$. The inequality of Lemma 2.5 implies that $c_{n}$ and $c_{n}^{*}$ are near each other in the Hausdorff metric and

$$
\ell\left(c_{n}\right)-\ell\left(c_{n}^{*}\right) \longrightarrow 0 .
$$

It follows from the algebraic convergence lemmas above that $\ell\left(c_{n}^{*}\right) \rightarrow \ell\left(c^{*}\right)$. By using inverses to the almost isometric embeddings $\psi_{n}$, it follows that $\lim \inf \ell\left(c_{n}\right)$ is at least $\ell(c)$. These facts can be combined to show $\ell(c)-\ell\left(c^{*}\right)=0$.

Lemma 5.13. Pick $\delta>0$. Define $K_{r, n}:=\cup_{i} B_{N_{n}}\left(\omega_{n}^{i}, r\right)$. There exists $r_{0}, n_{0}>0$ such that: if $n>n_{0}$ and $r>r_{0}$ then the volume of the set

$$
C_{N_{n}}-K_{r, n}
$$

is less than $\delta$. 
Proof: The main tool of the proof will be the isoperimetric inequality for hyperbolic manifolds with abelian fundamental group. Namely, if $H<\mathrm{PSl}_{2} \mathbb{C}$ is abelian and $Y \subset \mathbb{H}^{3} / H$ is a Lipschitz embedded finite volume 3-dimensional submanifold with boundary then

$$
\operatorname{Vol}(Y) \leq \operatorname{Area}(\partial Y) .
$$

This inequality follows immediately from the proof of [34, Prop.4.1].

Recall the constant $\eta_{0}$ from Proposition 5.11. Fix $\eta<\eta_{0}$ sufficiently small such that $C_{N} \cap N^{>\eta}$ is homeomorphic to $C_{N}$ via the obvious map which retracts the cusps onto the thick part, the area of the surface of intersection

$$
S:=C_{N} \cap \partial N^{\geq \eta}
$$

is less than $\delta / 4$, and the boundary of $S$ has total length less than $\delta / 4 . \quad S$ is a collection of annuli and tori. Note that $C_{N} \cap N^{<\eta}$ has totally geodesic boundary (see Section 2.3).

Pick $r_{0}>0$ such that $C_{N} \cap N^{\geq \eta} \subseteq K_{r_{0}}$. Let $r>r_{0}$.

Using the almost isometric embeddings given by geometric convergence, there is an $n_{0}$ such that $n>n_{0}$ implies the area of the intersection

$$
S_{n}:=C_{N_{n}} \cap \partial N_{n}^{\geq \eta} \cap K_{r, n}
$$

is less than $\delta / 2$, and the boundary of $S_{n}$ has total length less than $\delta / 2$. This estimate can be proven carefully by a packing argument, using the fact that the local geometry of $S_{n}$ is horospherical (i.e. the same for all $n$ ).

The manifold $(M, P)$ is "filled up" by the components of $N$ in the following sense: each nonelementary interval bundle in the characteristic submanifold of $(M, P)$ is formed by gluing up some components of $C_{N} \cap N \geq \eta$ along annuli, and each pared acylindrical component of the complement of the characteristic submanifold of $(M, P)$ is homeomorphic to a component of $C_{N}$. Since the maps $\psi_{n}$ are embeddings, for each $n$ the subsurface $\partial\left(C_{N_{n}} \cap N_{n}^{\leq \eta}\right)$ is formed from $S_{n}$ by successively performing one of the following two operations: joining two boundary components of $S_{n}$ by a ruled annulus (which lies in the boundary of the convex core $C_{N_{n}}$ ), or attaching to a single boundary component of $S_{n}$ a half-infinite ruled annulus (lying on the boundary of $C_{N_{n}}$ ) going out a rank one cusp of $N_{n}$.

A basic fact from hyperbolic geometry is that the area of a hyperbolic ruled annulus (not necessarily compact) is less than the length of its boundary [32, Thm.9.3.1]. Thus the area of $\partial\left(C_{N_{n}} \cap N_{n}^{\leq \eta}\right)$ is less than $\delta$. So finally, by the isoperimetric inequality, the volume of $\left(C_{N_{n}} \cap N_{n}^{\leq \eta}\right)$ is at most $\delta$.

From this it follows that $\operatorname{Vol}\left(C_{N_{n}}\right) \longrightarrow \operatorname{Vol}\left(C_{N}\right)$. Since each component of $C_{N}$ is either a totally geodesic surface or a 3-manifold with totally geodesic boundary, it follows from Proposition 2.7 that the volume of $C_{N}$ is exactly half the simplicial volume of the doubled manifold $D\left(\mathcal{N}_{1} C_{N} \cap N^{\geq \eta}, \mathcal{N}_{1} C_{N} \cap \partial N^{\geq \eta}\right)$ (where $\mathcal{N}_{1} C_{N}$ is a radius 1 regular neighborhood of $C_{N}$ and $\eta$ is the constant from the proof of Lemma 5.13). Since the manifold $D\left(\mathcal{N}_{1} C_{N} \cap N^{\geq \eta}, \mathcal{N}_{1} C_{N} \cap \partial N^{\geq \eta}\right)$ can be obtained topologically by cutting $D(M, P)$ along essential tori, it follows from Theorem 2.6 that they have the same simplicial volume. In particular, it follows that

$$
\operatorname{Vol}\left(C_{N_{n}}\right) \longrightarrow \frac{1}{2} \operatorname{SimpVol}(D(M, P))
$$

This completes the proof of Theorem 5.1 


\section{REFERENCES}

[1] I. Agol. Topology of Hyperbolic 3-Manifolds. Ph.D. thesis, University of California San Diego, 1998.

[2] J. Anderson and R. Canary. Cores of hyperbolic 3-manifolds and limits of Kleinian groups II. J. London Math. Soc., 61(2):489-505, 2000.

[3] R. Benedetti and C. Petronio. Lectures on Hyperbolic Geometry. Universitext. SpringerVerlag, 1992.

[4] L. Bessières. Sur le volume minimal des variétés ouvertes. Ann. Inst. Fourier, 50(3):965-980, 2000.

[5] G. Besson, G. Courtois, and S. Gallot. Entropies et rigidités des espaces localement symétriques de courbure strictement négative. Geom. Funct. Anal., 5(5):731-799, 1995.

[6] F. Bonahon. A Schläfli-type formula for convex cores of hyperbolic 3-manifolds. J. Diff. Geom., 50:25-58, 1998.

[7] F. Bonahon and J.-P. Otal. Laminations mesurées de plissage des variétés hyperboliques de dimension 3. Ann. Math., 160:1013-1055, 2004.

[8] B. Bowditch. Some results on the geometry of convex hulls in manifolds of pinched negative curvature. Comm. Math. Hel., 69(1):49-81, 1994.

[9] M. Bridgeman and R. Canary. From the boundary of the convex core to the conformal boundary. Geometriae Dedicata, 96:211-240, 2003.

[10] D. Burago, Y. Burago, and S. Ivanov. A Course in Metric Geometry, volume 33 of Graduate Studies in Mathematics. AMS, Providence, 2001.

[11] Y. Burago, M. Gromov, and G. Perel'man. A. D. Aleksandrov spaces with curvatures bounded below. Uspekhi Mat. Nauk. (Russian), Russian Math. Surveys (English), 47(2):3-51 (Russian), 1-58 (English), 1992.

[12] R. Canary. Covering theorems for hyperbolic 3-manifolds. In Proceedings of Low-Dimensional Topology, pages 21-30. International Press, 1994.

[13] R. Canary, Y. Minsky, and E. Taylor. Spectral theory, Hausdorff dimension and the topology of hyperbolic 3-manifolds. Journal of Geometric Analysis, 9:17-40, 1999.

[14] D. Epstein and A. Marden. Convex hulls in hyperbolic space, a theorem of Sullivan, and measured pleated surfaces. In Analytical and Geometric Aspects of Hyperbolic Spaces, pages 3-92. Cambridge University Press, Cambridge, 1987.

[15] C. Hodgson and S. Kerckhoff. Rigidity of hyperbolic cone-manifolds and hyperbolic Dehn surgery. J. Diff. Geom., 48:1-60, 1998.

[16] W. Jaco. Lectures on Three-Manifold Topology, volume 43 of CBMS Regional Conference Series in Mathematics. Amer. Math. Soc., Providence, R.I., 1980.

[17] W. Jaco and P. Shalen. Seifert fibered spaces in 3-manifolds, volume 220 of Memoirs of the Amer. Math. Soc. Amer. Math. Soc., Providence, RI, 1979.

[18] K. Johannson. Homotopy Equivalences of 3-manifolds with Boundary, volume 761. SpringerVerlag, Berlin and New York, 1979

[19] T. Jørgensen and A. Marden. Algebraic and geometric convergence of kleinian groups. Math. Scand., 66:47-72, 1990 .

[20] S. Kerckhoff and W. Thurston. Noncontinuity of the action of the modular group at Bers' boundary of Teichmüller space. Invent. Math., 100(1):25-47, 1990.

[21] C. Lecuire. Structures hyperboliques convexes sur les variétés de dimension 3. Ph.D. thesis, ENS Lyon, 2003.

[22] B. Leeb. 3-manifolds with(out) metrics of nonpositive curvature. Invent. Math., 122(2):277289, 1995.

[23] C. McMullen. Renormalization and 3-manifolds which fiber over the circle. Princeton University Press, Princeton, NJ, 1996.

[24] C. McMullen. Hausdorff dimension and conformal dynamics I: Kleinian groups and strong limits. J. Diff. Geom., 51:471-515, 1999.

[25] J. Morgan. On Thurston's uniformization theorem for three-dimensional manifolds. In J. Morgan and H. Bass, editors, The Smith Conjecture, pages 37-125. Academic Press, 1984.

[26] G. Perel'man. Alexandrov spaces with curvatures bounded from below II. Preprint, 1991.

[27] T. Soma. The Gromov invariant of links. Invent. Math., 64(3):445-454, 1981. 
[28] J. Souto. Geometric Structures on 3-Manifolds and their Deformations. Bonner mathematische schriften, Rheinischen-Friedrich-Wilhelms-Universität Bonn, 2001. Portions co-authored with J. Boland, C. Connell, and G. Kleineidam.

[29] P. Storm. Minimal volume Alexandrov spaces. J. Diff. Geom., 61(2):195-226, 2002.

[30] P. Storm. The barycenter method on singular spaces. Comment. Math. Helv., 82(1):133-173, 2007.

[31] E.C. Taylor. Geometric finiteness and the convergence of Kleinian groups. Comm. Anal. Geom., 5(3):497-533, 1997.

[32] W. Thurston. The topology and geometry of 3-manifolds. Available from the MSRI website www.msri.org, 1976-1979. Princeton Univ. lecture notes.

[33] W. Thurston. Hyperbolic geometry and 3-manifolds. In Low-dimensional Topology (Bangor, 1979), volume 48 of London Math. Soc. Lecture Note Ser., pages 9-25. Cambridge Univ. Press, 1982.

[34] W. Thurston. Hyperbolic structures on 3-manifolds I: Deformation of acylindrical manifolds. Ann. of Math., 124(2):203-246, 1986.

[35] W. Thurston. Hyperbolic structures on 3-manifolds, III: Deformations of 3-manifolds with incompressible boundary. Available at front.math.ucdavis.edu/math.GT/9801058, 1986. 